\title{
Charge redistribution in correlated heterostuctures within nonequilibrium real-space dynamical mean-field theory
}

\author{
Irakli Titvinidze, Max E. Sorantin, Antonius Dorda, Wolfgang von der Linden, and Enrico Arrigoni \\ Institute of Theoretical and Computational Physics, Graz University of Technology, 8010 Graz, Austria
}

(Received 15 May 2018; revised manuscript received 12 July 2018; published 27 July 2018)

\begin{abstract}
We address the steady-state behavior of a system consisting of several correlated monoatomic layers sandwiched between two metallic leads under the influence of a bias voltage. In particular, we investigate the interplay of the local Hubbard and the long-range Coulomb interaction on the charge redistribution at the interface, in the paramagnetic regime of the system. We provide a detailed study of the importance of the various system parameters, like Hubbard $U$, lead-correlated region coupling strength, and the applied voltage on the charge distribution in the correlated region and in the adjacent parts of the leads. In addition, we also present results for the steady-state current density and double occupancies. Our results indicate that, in a certain range of parameters, the charge on the two layers at the interface between the leads and the correlated region display opposite signs, producing a dipolelike layer at the interface. Our results are obtained within nonequilibrium (steady-state) real-space dynamical mean-field theory, with a self-consistent treatment of the long-range part of the Coulomb interaction by means of the Poisson equation. The latter is solved by the Newton-Raphson method and we find that this significantly reduces the computational cost compared to existing treatment. As the impurity solver for real-space dynamical mean-field theory, we use the auxiliary master equation approach, which addresses the impurity problem within a finite auxiliary system coupled to Markovian environments.
\end{abstract}

DOI: 10.1103/PhysRevB.98.035146

\section{INTRODUCTION}

Correlated systems out of equilibrium and especially electronic transport through heterostructures made from different materials have attracted increasing interest due to the recent impressive experimental progress to fabricate correlated heterostructures [1-6] with atomic resolution and, in particular, growing atomically abrupt layers with different electronic structures [1-3].

From a theoretical perspective, investigating and understanding the physical processes which govern the behavior of such systems is a great challenge in the field of theoretical solid state physics. For instance, it was shown that, due to the proximity effect, any finite number of Mott-insulating layers become metallic when sandwiched between semi-infinite metallic leads [7-14]. For such a geometry, the effect of impact ionization in periodically driven Mott-insulating layers was studied $[15,16]$ as well as resonance phenomena in a system consisting of several correlated and noncorrelated monoatomic layers [17]. Another challenging aspect of such systems that was investigated is the capacitance of multilayer systems made from correlated materials [18-20]. Due to the local Hubbard and long-range Coulomb interaction (LRCI) present in these systems, charge redistribution takes place [20-22]. The equilibrium situation was addressed, e.g., in Refs. [21,22]. In particular, Ref. [21] studied the charge redistribution and the corresponding thermoelectric properties for a metal, strongly correlated barrier-metal device where the on-site energies of the correlated region are shifted compared to the metals, while Ref. [22] investigated the behavior of the correlated thin film in a transverse electric field. Finally, Ref. [20] considered correlated layers described by the Falicov-Kimball model, where one spin-species is immobile, with emphasis on the nonequilibrium situation arising due to an applied bias voltage.

Here, we investigate a system of correlated layers sandwiched between two metallic leads in the paramagnetic phase, see Fig. 1 for an illustration. Similar to Ref. [20], we take into account LRCIs, but here we use the Hubbard model where both spin-species are mobile. The goal of the current paper is to investigate the influence of local Hubbard and LRCIs on the charge redistribution in a nonequilibrium steady-state situation produced by an applied bias voltage.

We obtain that the charge density deviation from the bulk filling on opposite sites of the lead-correlated (LC) junction have opposite signs in a certain range of parameters, indicating the formation of a dipolelike layer. According to our calculations, such a layer arises for small values of the hybridisation $t_{\mathrm{lc}}$ at the LC junction for all considered interactions and bias voltages. On the other hand, for large $t_{\mathrm{lc}}$ it occurs only for weak to intermediate interactions and at low bias voltages.

To describe the behavior of the system, we adopt dynamical mean-field theory (DMFT) [23-25], which is one of the most powerful methods to investigate high-dimensional strongly correlated electron systems. DMFT was originally developed to describe translationally invariant systems in equilibrium, but was later extended to inhomogeneous systems $[7,8,12$ $14,16,17,26-55]$, and also adapted to the nonequilibrium case $[12,13,56-64]$. In the latter, DMFT is formulated within the nonequilibrium Green's function approach originating from the works of Kubo [65], Schwinger [66], Kadanoff and Baym [67,68], and Keldysh [69]. The only approximation in DMFT is the assumption of a local self-energy. This can be calculated by mapping the original problem onto a single impurity Anderson model (SIAM) [70], whose parameters are determined 


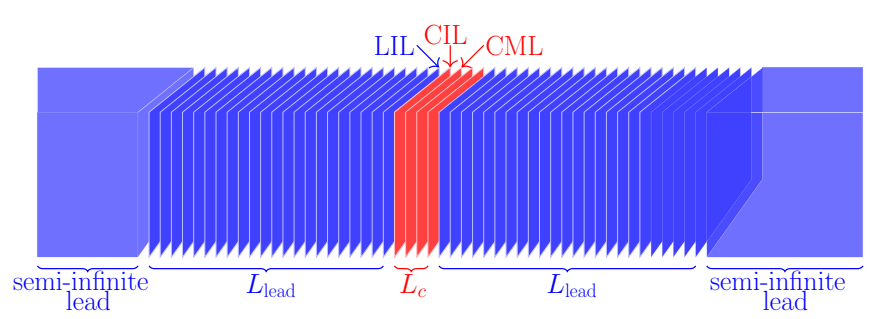

FIG. 1. A Schematic representation of the system consisting of $L_{c}=4$ correlated interfaces (red) sandwiched between two semiinfinite metallic leads (blue). In addition to the local Hubbard interaction, present only within the correlated layers, we also take into account long-range Coulomb forces extending into the leads. We take them into account by solving the Poisson equation in an extended region including part of the lead layers ( $L_{\text {lead }}=23$ for each side). Here LIL, CIL, and CML stand for lead interface layer, correlated interface layer, and correlated middle layer, respectively.

self-consistently. For homogeneous systems, the self-energy is the same for each lattice site due to translational symmetry, and thus one needs to solve only one SIAM problem, per DMFT iteration, while for systems with broken translational invariance, such as the one considered here, one needs to solve many impurity problems to capture the spatial inhomogeneity of the system. In the current work, the nonequilibrium SIAM problems are solved by using the recently developed auxiliary master equation approach (AMEA) [62,63,71], which treats the impurity problem within an auxiliary system consisting of a correlated impurity, a small number $N_{B}$ of uncorrelated bath sites and two Markovian environments described by a Lindblad master equation. The approach allows for an accurate solution of the steady-state impurity problem already with a small $N_{B}$.

For the self-consistent solution of the nonlinear Poisson equation, we used the Newton-Ralphson, which significantly improves the convergence.

The paper is organized as follows. Section II decribes the model and method. In particular, in Sec. II A we introduce the Hamiltonian of the system, in Sec. IIB we illustrate the application of real-space DMFT within the nonequilibrium steady-state Green's function formalism for a system consisting of many layers, in Sec. IIC we give an overview of the solution of the Poisson equation and, finally, in Sec. IID we present the self-consistency loop used to obtain the self-consistent results. Thereafter, in Sec. III we present our results, and our conclusions are presented in Sec. IV.

\section{MODEL AND METHOD}

\section{A. Model}

We consider a system consisting of a correlated region (c) with $L_{c}$ correlated infinite and translationally invariant layers attached to two metallic leads $(\alpha=l, r)$, which are semi-infinite in the $z$ direction and translationally invariant in the $x y$ plane (parallel to the correlated layers). The physical situation is depicted in Fig. 1 and described by the
Hamiltonian

$$
\begin{aligned}
\mathcal{H}= & -\sum_{z,\left\langle\mathbf{r}, \mathbf{r}^{\prime}\right\rangle, \sigma} t_{z} c_{z, \mathbf{r}, \sigma}^{\dagger} c_{z, \mathbf{r}^{\prime}, \sigma}-\sum_{\left\langle z, z^{\prime}\right\rangle, \mathbf{r}, \sigma} t_{z z^{\prime}} c_{z, \mathbf{r}, \sigma}^{\dagger} c_{z^{\prime}, \mathbf{r}, \sigma} \\
& +\sum_{z, \mathbf{r}} U_{z} n_{z, \mathbf{r}, \uparrow} n_{z, \mathbf{r}, \downarrow}+\sum_{z, \mathbf{r}, \sigma}\left(v_{z}^{(0)}+v_{z}\right) n_{z, \mathbf{r}, \sigma}
\end{aligned}
$$

Here $c_{z, \mathbf{r}, \sigma}^{\dagger}$ creates an electron at site $\mathbf{r}=(x, y)$ of layer $z$ with spin $\sigma$ and $n_{z, \mathbf{r}, \sigma}=c_{z, \mathbf{r}, \sigma}^{\dagger} c_{z, \mathbf{r}, \sigma}$ denotes the corresponding occupation-number operator. $\left\langle z, z^{\prime}\right\rangle$ stands for neighboring $z$ and $z^{\prime}$ layers and $\left\langle\mathbf{r}, \mathbf{r}^{\prime}\right\rangle$ stands for neighboring $\mathbf{r}$ and $\mathbf{r}^{\prime}$ sites in the same layer.

The first two terms of the Hamiltonian Eq. (1) describe nearest-neighbor intralayer and interlayer hoppings, with hopping amplitudes $t_{z}$ and $t_{z z^{\prime}}$, respectively. The third term introduces the local Hubbard interactions $U_{z}$, which are nonzero only for the correlated region. The last term describes the on-site energies, whereby $v_{z}^{(0)}$ is chosen such that we obtain the required bulk filling in the $z$ th layer with the special case of $v_{z}^{(0)}=-U_{z} / 2$ at half-filling $(\mathrm{HF})$. Furthermore, $v_{z}$ describes the Hartree shift of the on-site energies obtained after the mean-field decoupling of the LRCI, $V_{i j}\left(n_{i}-1\right)\left(n_{j}-1\right)$, which is produced by the charge inhomogeneity and has to be determined self-consistently. In contrast to the local Hubbard interaction, the LRCI affects not only the correlated region, but the leads as well. Therefore, we incorporate parts of the leads, namely $L_{\text {lead }}$ layers per side, into the region. Here, $L_{\text {lead }}$ has to be chosen large enough such that the (self-consistently determined) electron density $v_{z}$ converges to the bulk filling of the leads far away from the correlated region. To summarize, the extended central region contains $L=L_{c}+2 L_{\text {lead }}$ layers. The corresponding indices vary from $-\frac{L-1}{2}$ to $\frac{L-1}{2}$. $|z|<L_{c} / 2$ describes the correlated region, $L_{c} / 2<|z|<L_{c} / 2+L_{\text {lead }}$ corresponds to the left $(z<0)$ and right $(z>0)$ leads, which we treat explicitly, while $|z|>L_{c} / 2+L_{\text {lead }}$ corresponds to the semi-infinite lead layers $(z<0$ left lead and $z>0$ right lead). Here we note that the chosen labeling convention leads to half-integer indices for even $L_{c}$ considered throughout the paper.

We take the Hubbard interaction to be uniform within the correlated region, i.e., $U_{z}=U$ for $|z|<L_{c} / 2$ and $U_{z}=0$ on the lead layers $\left(|z|>L_{c} / 2\right)$. We assume isotropic nearestneighbor hopping parameters within the correlated region as well as in the leads, respectively. This amounts to the choice $t_{z z^{\prime}}=t_{z} \equiv t_{c}$ for the correlated region $\left(|z|<L_{c} / 2\right)$ and $t_{z z^{\prime}}=t_{z} \equiv t_{\alpha=l, r}$ for the leads $\left(|z|>L_{c} / 2\right)$. Finally, the lead-correlated region junction (LC-junction) coupling is the same on both sides $t_{-\frac{L_{c}+1}{2},-\frac{L_{c}-1}{2}}=t_{\frac{L_{c}-1}{2}, \frac{L_{c}+1}{2}} \equiv t_{\mathrm{lc}}$. We work in units where $e=\hbar=k_{b}^{2}=a^{2}=1$, with $a^{2}$ denoting the lattice spacing and take $t_{c}=1$ as unit of energy.

The nonequilibrium situation is reached by applying a bias voltage $V=v_{l}-v_{r}$. Here $v_{l}$ and $v_{r}$ are the on-site energies far away from the correlated region $\left(v_{l / r}=v_{z= \pm \infty}\right)$. Notice that, in general, $V$ is not equal to the difference between the chemical potentials of the leads $\Delta \mu=\mu_{l}-\mu_{r}$ due to the contribution from back-scattered electrons [72].

To investigate steady-state properties of our system, we work within the Keldysh Green's function formalism $[66,68,69,73,74]$ and use real-space dynamical mean-field 
theory (R-DMFT) combined with the Poisson equation to treat the Hubbard interaction and long-range coulomb forces, respectively.

\section{B. Real-space dynamical mean-field theory}

Here, we give only a brief overview of the nonequilibrium real-space DMFT approach [12,13,17,57-61] together with the employed impurity solver, namely the auxiliary master exquation approach (AMEA) [62,63,71,75].

In the nonequilibrium situation, the model remains translationally invariant along the $x y$ plane (parallel to the layers), which allows us to introduce the corresponding momenta $\mathbf{k}=$ $\left(k_{x}, k_{y}\right)$. Moreover, since the steady-state Green's functions depend only on the time difference, it is convenient to transform them to the frequency domain $\omega$.

The Green's function for the extended central region, which consists of $L=L_{c}+2 L_{\text {lead }}$ layers, can be expressed via Dyson's equation:

$$
\left[\mathbf{G}^{-\mathbf{1}}\right]^{\gamma}(\omega, \mathbf{k})=\left[\mathbf{G}_{0}^{-1}(\omega, \mathbf{k})\right]^{\gamma}-\boldsymbol{\Sigma}^{\gamma}(\omega)
$$

Here, boldface indicates $L \times L$ matrices, while $\gamma$ stands for retarded (R), advanced (A), and Keldysh (K) components. $\mathbf{G}^{A}$ and $\mathbf{G}^{R}$ are related via $\mathbf{G}^{A}=\left(\mathbf{G}^{R}\right)^{\dagger}$, while $\mathbf{G}^{K}$, in general, is independent of $\mathbf{G}^{R}$ and needs to be determined separately.

The inverse of the noninteracting Green's function reads

$$
\begin{gathered}
{\left[\mathbf{G}_{\mathbf{0}}^{-\mathbf{1}}\right]_{z z^{\prime}}^{R}(\omega, \mathbf{k})=t_{z z^{\prime}}+\delta_{z z^{\prime}}\left(\omega-v_{z}-v_{z}^{(0)}-E_{z}(\mathbf{k})\right)} \\
-\delta_{z z^{\prime}} \Sigma_{\mathrm{hyb}, z}^{R}(\omega, \mathbf{k}), \\
{\left[\mathbf{G}_{\mathbf{0}}^{-\mathbf{1}}\right]_{z z^{\prime}}^{K}(\omega, \mathbf{k})=-\delta_{z z^{\prime}} \Sigma_{\mathrm{hyb}, z}^{K}(\omega, \mathbf{k}),}
\end{gathered}
$$

where $E_{z}(\mathbf{k})$ is the dispersion relation for the $z$ th layer of the the extended central region and

$$
\Sigma_{\text {hyb }, z}^{\gamma}(\omega, \mathbf{k})=\delta_{z,-\frac{L-1}{2}} t_{l}^{2} g_{l}^{\gamma}(\omega, \mathbf{k})+\delta_{z, \frac{L-1}{2}} t_{r}^{2} g_{r}^{\gamma}(\omega, \mathbf{k})
$$

describes the hybridization between the semi-infinite leads and the extended central region. $g_{l}^{\gamma}(\omega, \mathbf{k})$ and $g_{r}^{\gamma}(\omega, \mathbf{k})$ denote the Green's functions for the interface layers of the semi-infinite leads disconnected from the extended central region. Their retarded component can be expressed as $[26,27,76]$

$$
\begin{aligned}
g_{\alpha}^{R}(\omega, \mathbf{k})= & \frac{\omega-v_{\alpha}-v_{\alpha}^{(0)}-E_{\alpha}(\mathbf{k})}{2 t_{\alpha}^{2}} \\
& -i \frac{\sqrt{4 t_{\alpha}^{2}-\left(\omega-v_{\alpha}-v_{\alpha}^{(0)}-E_{\alpha}(\mathbf{k})\right)^{2}}}{2 t_{\alpha}^{2}},
\end{aligned}
$$

where $v_{\alpha=l / r}+v_{\alpha=l / r}^{(0)}$ and $E_{\alpha=l / r}(\mathbf{k})$ denote the on-site energies and the dispersion relation for the left/right lead, respectively. The sign of the square-root for negative argument in Eq. (6) must be chosen such that the Green's function has the correct $1 / \omega$ behavior for $|\omega| \rightarrow \infty$. Since the disconnected leads are separately in equilibrium, we can obtain their Keldysh components from the retarded ones via the fluctuation dissipation theorem [73]:

$$
g_{\alpha}^{K}(\omega, \mathbf{k})=2 i\left(1-2 f_{\alpha}(\omega)\right) \operatorname{Im} g_{\alpha}^{R}(\omega, \mathbf{k}) .
$$

Here, $f_{\alpha}(\omega)$ is the Fermi distribution for the chemical potential $\mu_{\alpha}$ and temperature $T_{\alpha}$.
Finally, $\boldsymbol{\Sigma}_{z z^{\prime}}^{\gamma}(\omega)=\delta_{z z^{\prime}} \Sigma_{z}^{\gamma}(\omega)$ stands for the self-energy matrix, which, due to the DMFT approximation, is diagonal and $\mathbf{k}$-independent. To determine it, we map each correlated layer $z$ to a (nonequilibrium) single impurity problem (SIAM) with Hubbard interaction $U_{z}$ and on-site energy $v_{z}+v_{z}^{(0)}$, coupled to a self-consistently determined bath. The latter is specified by its hybridization function obtained as (see, e.g., Refs. [17,24])

$$
\begin{gathered}
\Delta_{z}^{R}(\omega)=\omega-v_{z}-v_{z}^{(0)}-\Sigma_{z}^{R}(\omega)-\frac{1}{G_{\mathrm{loc}, z}^{R}(\omega)}, \\
\Delta_{z}^{K}(\omega)=-\Sigma_{z}^{K}(\omega)+\frac{G_{\mathrm{loc}, z}^{K}(\omega)}{\left|G_{\mathrm{loc}, z}^{R}(\omega)\right|^{2}},
\end{gathered}
$$

where the local Green's function is defined as

$$
G_{\mathrm{loc}, z}^{\gamma}(\omega)=\int_{\mathrm{BZ}} \frac{d^{2} \mathbf{k}}{(2 \pi)^{2}} \mathbf{G}_{z z}^{\gamma}(\omega, \mathbf{k}) .
$$

To calculate the diagonal elements of the matrices $\mathbf{G}^{\gamma}(\omega, \mathbf{k})$ from Eq. (2), we use the recursive Green's function method $[16,17,77,78]$, which we generalize to the present situation of Keldysh Green's functions [17].

To describe the lattice structure of the isolated layers, we use a Bethe-lattice density of state (DOS). Due to this choice, we can replace $E_{z}(\boldsymbol{k})$ by $t_{z} \varepsilon$ and $\int \frac{d \boldsymbol{k}}{(2 \pi)^{2}}$ by $\int d \varepsilon \rho(\varepsilon)$, where $\varepsilon$ is a dimensionless parameter characterizing the energy and $\rho(\varepsilon)=\frac{1}{\pi} \sqrt{4-\varepsilon^{2}}$ is the Bethe-lattice DOS.

The corresponding impurity problems are then solved with AMEA, which is a state-of-the-art impurity solver particularly suited to address the steady state. AMEA is based upon mapping $[62,75]$ the SIAM to an open quantum system of finite size, which includes one correlated site, $N_{B}$ noninteracting bath sites and two Markovian environments, whose dynamics is governed by a Lindblad master equation. The resulting open quantum system can then be solved by numerical manybody techniques such as Krylov-space-based [63,71] methods (which are the ones we use here), matrix product states (MPS) [79], or the so-called stochastic wave function algorithm $[80,81]$.

\section{Charge reconstruction}

To take into account long-range Coulomb forces on a meanfield level, we calculate the on-site energies $v_{z}$ self-consistently by solving the corresponding Poisson equation:

$$
\frac{\partial}{\partial z}\left(\frac{1}{c_{z}} \frac{\partial v_{z}}{\partial z}\right)=-\left(n_{z}-n^{\text {bulk }}\right) .
$$

It is convenient to adopt von Neumann boundary conditions, which in discretized form amounts to setting the Coulomb potential of the two bulk semi-infinite leads equal to the one of the boundary layers of the extended central region:

$$
v_{l / r}=v_{\mp \frac{L-1}{2}} .
$$

Here $c_{z} \equiv \frac{1}{\varepsilon_{0} \varepsilon_{r, z}}, \varepsilon_{r, z}$ is the relative permittivity of layer $z$ and $\varepsilon_{0}$ is the permittivity of free space. Moreover,

$$
n_{z}=1+\frac{1}{2 \pi} \int_{-\infty}^{\infty} d \omega \Im m G_{\mathrm{loc}, z}^{K}(\omega)
$$


is the electron density at layer $z$ obtained from nonequilibrium R-DMFT and $n^{\text {bulk }}$ is the bulk electron density, which we set equal to $1(\mathrm{HF})$ throughout this paper [82].

One way to proceed would be to fix the bias voltage $V$ and in the present particle-hole symmetric case $v_{l}=-v_{r}=V / 2$. In this case, one should adjust the asymptotic chemical potentials $\mu_{l}$ and $\mu_{r}$ of the leads to obtain the correct asymptotic charge neutrality $n_{z \rightarrow \pm \infty} \rightarrow n^{\text {bulk }}=1$. This is numerically demanding. Another alternative is to carry out the calculations for given $\mu_{l}=-\mu_{r}=\Delta \mu / 2$ and update the values of the on-site energies in the semi-infinite leads after each iteration, according to Eq. (12). The bias voltage is then determined by $V=v_{l}-v_{r}$ a posteriori. Here we follow the second strategy as it is numerically more convenient. In fact, we find that the difference between $\Delta \mu$ and $V$ is quite small in most of the calculations presented in this paper ( $1 \%$ or smaller), except for weak to intermediate $U$ at large $t_{l c}$, as we will discuss below.

For better readability, we introduce a vector notation for the $z$-dependent quantities, namely,

$$
\begin{aligned}
\vec{v} & =\left\{v_{-\frac{L-1}{2}}, \ldots, v_{\frac{L-1}{2}}\right\}, \\
\vec{n} & =\left\{n_{-\frac{L-1}{2}}, \ldots, n_{\frac{L-1}{2}}\right\}, \\
\vec{G}_{\mathrm{loc}}(\omega) & =\left\{G_{\mathrm{loc},-\frac{L-1}{2}}^{R}, \ldots, G_{\mathrm{loc}, \frac{L-1}{2}}^{R}, G_{\mathrm{loc},-\frac{L-1}{2}}^{K}, \ldots, G_{\mathrm{loc}, \frac{L-1}{2}}^{K}\right\}, \\
\vec{\Sigma}(\omega) & =\left\{\Sigma_{-\frac{L-1}{2}}^{R}, \ldots, \Sigma_{\frac{L-1}{2}}^{R}, \Sigma_{-\frac{L-1}{2}}^{K}, \ldots, \Sigma_{\frac{L-1}{2}}^{K}\right\} .
\end{aligned}
$$

Obviously, the elements of $\vec{\Sigma}$ are zero outside of the correlated region.

The electron densities depend, through $G_{\mathrm{loc}, z}^{K}(\omega)$ in Eq. (13), on the on-site energies as well as on the self-energy. The self-energy in turn is, through the self-consistency in R-DMFT, a functional of the on-site energies and of itself, i.e., $\vec{\Sigma}=$ $\vec{\Sigma}(\vec{v}, \vec{\Sigma})$. Thus, we have to solve Eqs. (11)-(13) together with the R-DMFT equations in a self-consistent manner.

For a fixed self-energy $\vec{\Sigma}(\omega)$, we solve Eqs. (11)-(13) by formulating it as a root searching problem, which we treat by the Newton-Raphson method. To this end, we define the function

$$
\Phi_{z}(\vec{v})=c_{z}\left[\frac{\partial}{\partial z}\left(\frac{1}{c_{z}} \frac{\partial v_{z}}{\partial z}\right)+\left(n_{z}(\vec{v}, \vec{\Sigma})-n^{\text {bulk }}\right)\right],
$$

of which we seek the zero. Following the Newton-Raphson scheme, we expand

$$
\Phi_{j}(\vec{v}+\Delta \vec{v})=\Phi_{j}(\vec{v})+\sum_{i} \frac{\partial \Phi_{j}(\vec{v})}{\partial v_{i}} \Delta v_{i} .
$$

Here $\Delta \vec{v}=\vec{v}^{(n+1)}-\vec{v}^{(n)}$ is the difference between two consecutive iterations in the self-consistent Poisson loop. Assuming $\Phi_{z}(\vec{v}+\Delta \vec{v}) \stackrel{!}{=} 0$, one obtains the following iteration scheme:

$$
\vec{v}^{(n+1)}=\vec{v}^{(n)}-M^{-1} \vec{\Phi}(\vec{v}),
$$

with $\vec{\Phi}=\left\{\Phi_{-\frac{L-1}{2}}, \ldots, \Phi_{\frac{L-1}{2}}\right\}$ and

$$
M_{j i}=\frac{\partial}{\partial v_{i}} \Phi_{j}(\vec{v}) .
$$

For the technical details about the discretization of the Poisson equation and the expression for the matrix elements $M_{i j}$, we refer to the Appendix.

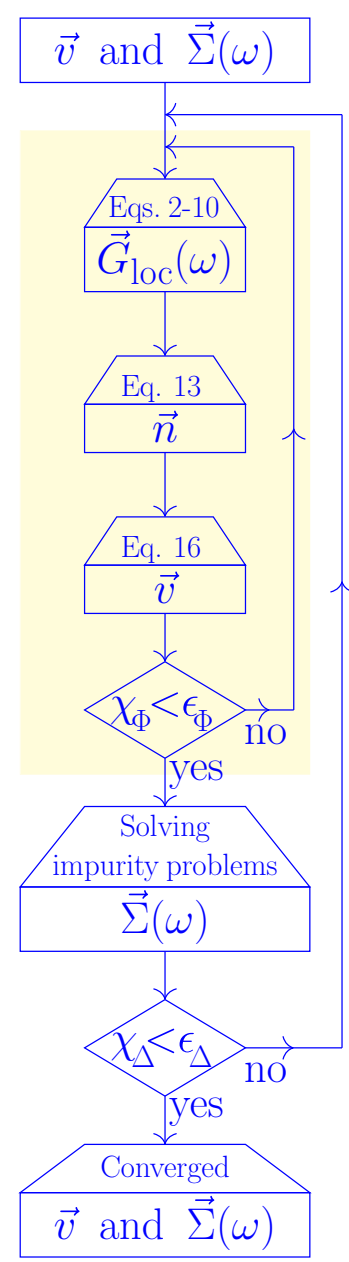

FIG. 2. A visualization of the self-consistency loop. The shaded area corresponds to the Poisson loop and we use the vector notation for $z$-dependent quantities, $\vec{v}, \vec{n}, \vec{\Sigma}(\omega)$ and $\vec{G}_{\text {loc }}(\omega)$ introduced in Sec. IIC. Moreover, $\chi_{\Phi}$ and $\chi_{\Delta}$ are cost functions for the convergence criteria defined in Eqs. (18) and (19), respectively.

\section{Self-consistency loop}

Here, we describe the self-consistency loop used to determine the self-energies $\vec{\Sigma}(\omega)$ together with the on-site energies $\vec{v}$ as self-consistent solution to the R-DMFT equations coupled, through the electronic number densities $\vec{n}(\vec{v}, \vec{\Sigma}(\omega))$, with the Poisson equation, Eq. (11). An illustration of the algorithm is presented in Fig. 2. In short, the iterative solution of the Poisson equation constitutes an inner loop to the R-DMFT selfconsistency and is done for fixed self-energies $\vec{\Sigma}(\omega)$ before the determination and solution of the impurity problems, which is more time demanding.

In more detail, we start with an initial guess of the selfenergies $\vec{\Sigma}(\omega)$ and on-site energies $\vec{v}$. Next, the Poisson loop is performed by calculating the electronic densities $\vec{n}$, Eq. (13), and updating the on-site energies according to Eq. (16). These two steps are then iterated until convergence [83] is reached, for which we require

$$
\chi_{\Phi} \equiv \sqrt{\frac{1}{L} \sum_{i} \Phi_{i}^{2}} \leqslant \epsilon_{\Phi},
$$




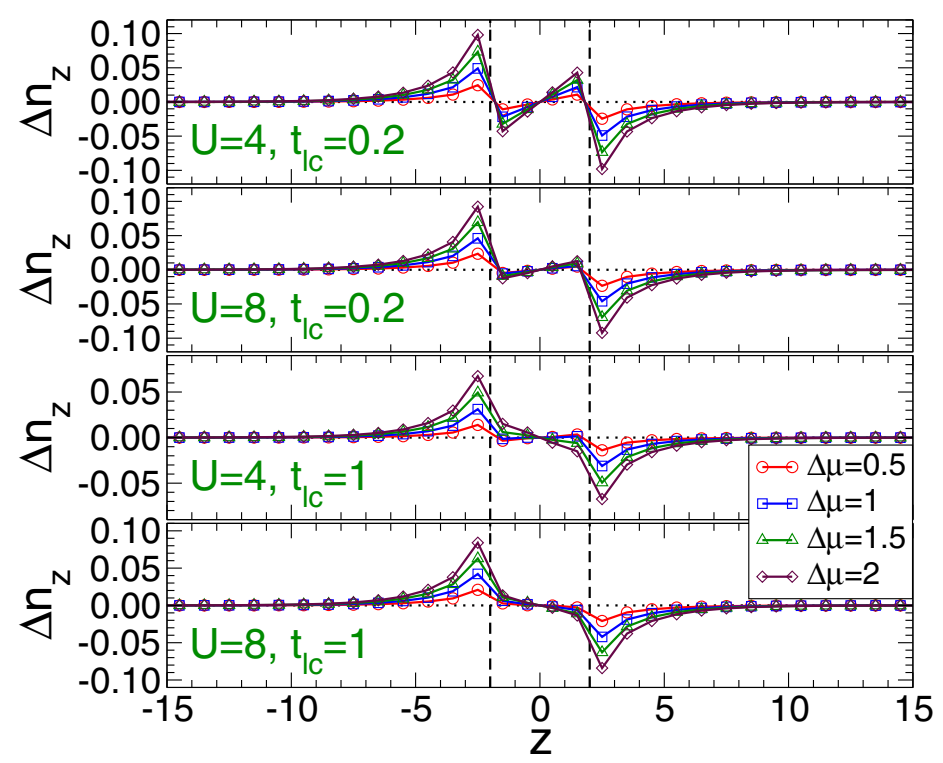

(a)

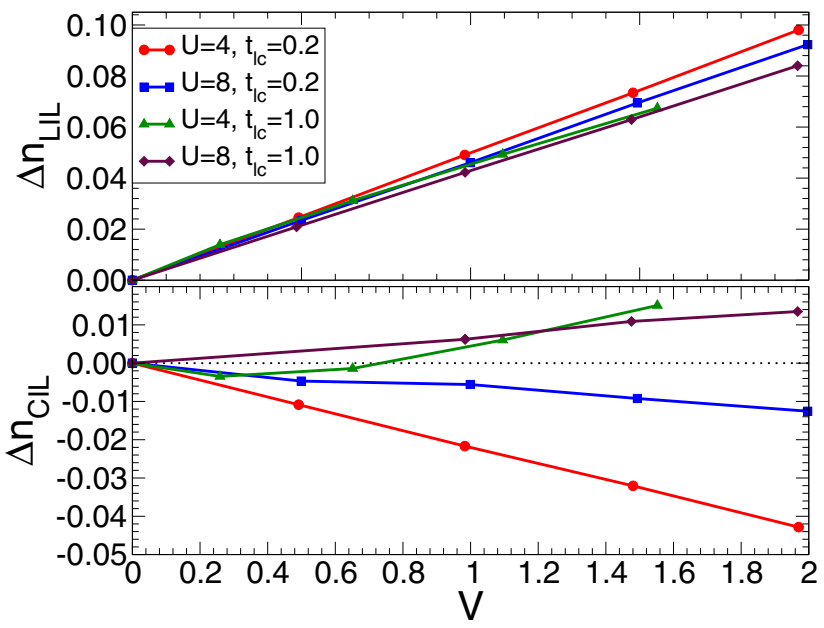

(b)

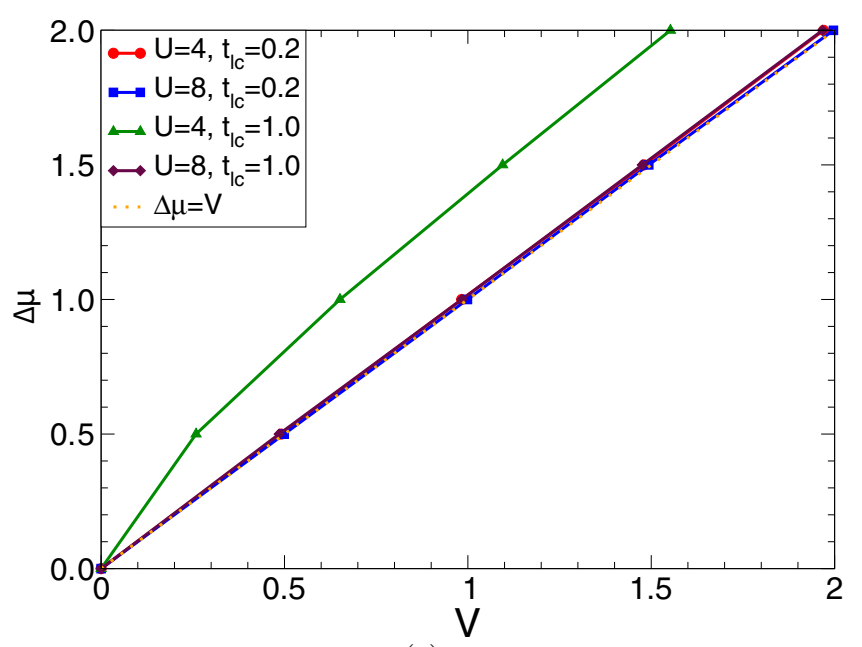

(c)

FIG. 3. Charge-density deviation from half-filling $\Delta n_{z}=n_{z}-1$ (a) as a function of layer index $z$ for Hubbard interactions $U=4$, 8 , LC-junction coupling $t_{\mathrm{lc}}=t_{\mathrm{rc}}=0.2,1$, and for different values of $\Delta \mu=\mu_{l}-\mu_{r}$. We present results for $L_{c}=4$ correlated layers. Other parameters are $t_{c}=1, t_{l}=t_{r}=2, v_{z}^{(0)}=-U_{z} / 2$, and $c=1.5$. The black dashed lines separate the correlated region and leads. (b) Upper panel $\Delta n_{z}$ for the lead interface layer (LIL) and lower panel $\Delta n_{z}$ for the correlated region interface layer (CIL) as a function of the bias voltage $V=v_{l}-v_{r}$. (c) Dependence of $\Delta \mu$ on the bias voltage $V$.

where $\epsilon_{\Phi}$ is the required accuracy. For each converged Poisson loop, we proceed with the corresponding on-site energies $\vec{v}$ to the R-DMFT iteration, which consists of computing the bath hybridization functions, Eqs. (8)-(9), and solving the corresponding impurity problems thereby obtaining a new set of self-energies $\vec{\Sigma}(\omega)$. The alternate solution of the Poisson equation and the impurity problems are then iterated until convergence of the R-DMFT loop. We quantify the accuracy of the latter by the weighted difference between the hybridization functions of two consecutive loops [84]:

$$
\chi_{\Delta} \equiv \frac{1}{L_{c}} \sqrt{\sum_{i=L_{\mathrm{lead}}+1}^{L_{\mathrm{lead}}+L_{c}} \int_{-\omega_{c}}^{\omega_{c}}\left\|\Delta_{i}^{(m)}-\Delta_{i}^{(m-1)}\right\| d \omega} \leqslant \epsilon_{\Delta},
$$

with

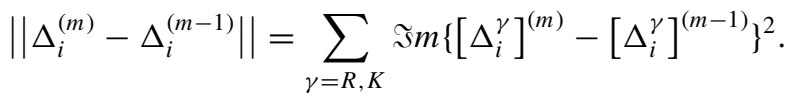

\section{RESULTS}

As mentioned in the introduction, the emphasis of the present work lies on the influence of electronic correlations on the charge redistribution in a nonequilibrium situation. To this end, we consider the heterostructure sketched in Fig. 1, which is driven out of equilibrium by an applied bias voltage.

To understand the behavior of the charge distribution, for finite $\mathrm{LC}$-junction coupling $\left(t_{\mathrm{lc}}>0\right)$ it is instructive to begin 
with a qualitative discussion of the expected behavior in the limit in which the correlated region is isolated from the leads $\left(t_{\mathrm{lc}}=0\right)$, but still capacitively coupled to them via the LRCI. In that case, when the correlated region is metallic, i.e., for weak to intermediate Hubbard interactions, the system consists of two capacitors (one at each LC-junction) connected in series. On the other hand, when the correlated region is insulating, i.e., for large values of the Hubbard interaction, it can be viewed as one capacitor with a dielectric material placed between two conducting materials. Applying a bias voltage will cause in both cases opposite charging of the facing surface layers of the lead and the correlated region, which can be viewed as dipolelike layers. For definiteness, we will refer to them as lead interface layer (LIL) and the correlated interface layer (CIL), respectively (see Fig. 1).

We perform calculations for $L_{c}=4$ and $L_{c}=40$ correlated layers, with a homogeneous local Hubbard interaction $U_{z}=$ $U$. For $L_{c}=4\left(L_{c}=40\right)$, we explicitly consider $L_{\text {lead }}=23$ $\left(L_{\text {lead }}=30\right)$ noninteracting, $U_{z}=0$, layers for each lead, to allow for proper charge redistribution in the leads as well. Therefore, in total, the extended central region, where the LRCI is accounted for, contains $L=50(L=100)$ layers. The infinite region outside of this range is treated exactly, whereby we take the charge and the Coulomb potential to be equal to its asymptotic bulk values. This is justified, as can be seen from Figs. 3(a), 4(a), 5(a), 5(b), 7(a), and 7(b). To work at particle-hole symmetry, we set the bare on-site energies $v_{z}^{(0)}=$ $-U_{z} / 2$ and the asymptotic lead charge densities $n_{z= \pm \infty}=1$. The hopping between nearest-neighbor correlated region sites is taken as unit of energy, $t_{c}=1$, and the hopping between nearest-neighbor sites of the leads is $t_{l}=t_{r}=2$. Further, to investigate the effect of the coupling strength of LC-junction on the behavior of the system, we perform calculations for different values of $t_{\mathrm{lc}}=0.2,0.4, \ldots, 1$. All calculations are performed at ambient temperature $T_{l}=T_{r}=0.025$ and we consider an isotropic Coulomb parameter with the moderate value $c_{z}=c=1.5$.

Due to particle-hole symmetry, properties of the $z$ th and $(-z)$ th layer are connected by a particle-hole transformation.

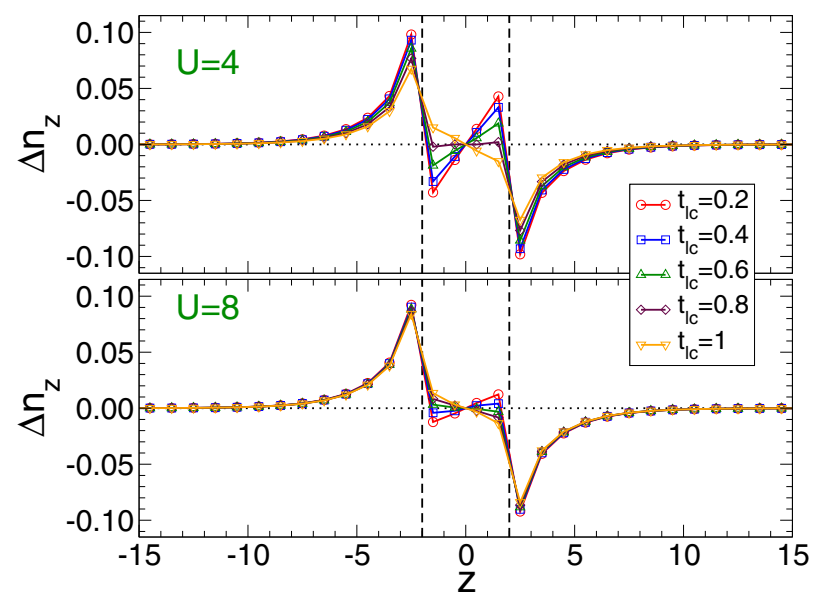

(a)
For the self-energies, the relation reads

$$
\begin{gathered}
\Sigma_{z}^{R}(\omega)=-\left[\Sigma_{-z}^{R}(-\omega)\right]^{*}+U_{z}, \\
\Sigma_{z}^{K}(\omega)=\left[\Sigma_{-z}^{K}(-\omega)\right]^{*} .
\end{gathered}
$$

Consequently, we need to calculate the self-energies only for half the system, i.e., $z<0$. Finally, all results for $L_{c}=4$ are obtained with $N_{b}=6$ bath sites in the AMEA, while for $L_{c}=$ 40 we considered $N_{B}=4$ due to the increased numerical effort [85].

\section{A. Effect of the bias voltage}

First, we investigate the effect of an applied bias voltage for intermediate, $U=4$, and strong, $U=8$, Hubbard interaction, as well as small $\left(t_{\mathrm{lc}}=0.2\right)$ and large $\left(t_{\mathrm{lc}}=1\right)$ coupling strengths between the leads and the correlated region.

Our calculations show that at the LC-junction, the system still hosts dipolelike layers for small but nonzero LC-junction coupling strengths. Figure 3(a) indeed shows for $t_{\mathrm{lc}}=0.2$, that the charge density deviations from HF, $\Delta n_{z}=n_{z}-1$, for the CIL and the LIL have opposite signs and their absolute values increase with bias voltage $V$ [see Fig. 3(b)] for both considered Hubbard interactions. So, similar to $t_{\mathrm{lc}}=0$, also for $t_{\mathrm{lc}}=0.2$, LIL and CIL can be viewed as dipolelike layers.

On the other hand, the behavior is qualitatively different for large values of the LC-junction coupling $\left(t_{\mathrm{lc}}=1\right)$ and, in particular, sensitive to the value of the Hubbard interaction. For strong interaction $(U=8)$, we obtain that $\Delta n_{z}$ of the LIL and CIL have the same sign and their absolute values increase with the bias voltage. When considering a weaker interaction $(U=4)$, this stays true for $\Delta n_{\text {LIL }}$ (charge density deviation from HF for the LIL), while $\Delta n_{\mathrm{CIL}}$ (charge density deviation from HF for the CIL) shows nonmonotonic behavior and a sign change as a function of the bias voltage. So, in contrast to small values of the LC-junction coupling strength, for large ones, dipolelike layers are only present at the LC-junction for weak to intermediate $U$ and low bias voltages.

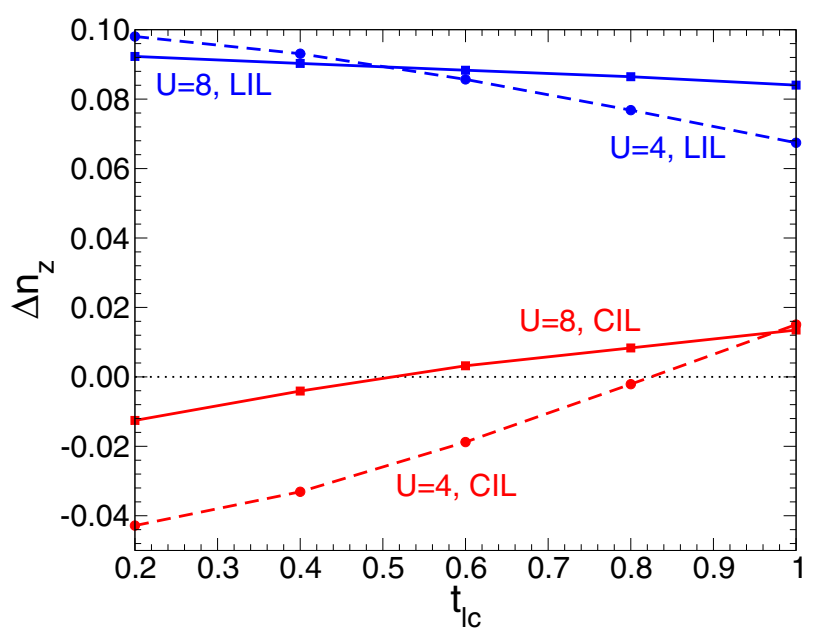

(b)

FIG. 4. (a) $\Delta n_{z}$ as a function of layer index $z$ for $U=4,8, \Delta \mu=2$, and different values of $t_{\mathrm{lc}}$. (b) $\Delta n_{z}$ for the LIL (blue curves) and CIL (red curves) as a function of the LC-junction coupling strength $t_{\mathrm{lc}}$. Other parameters are the same as in Fig. 3. 


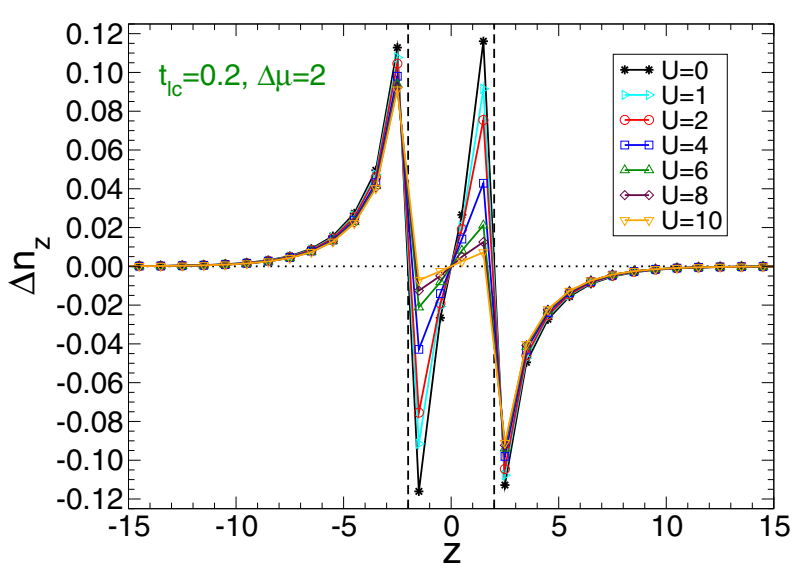

(a)

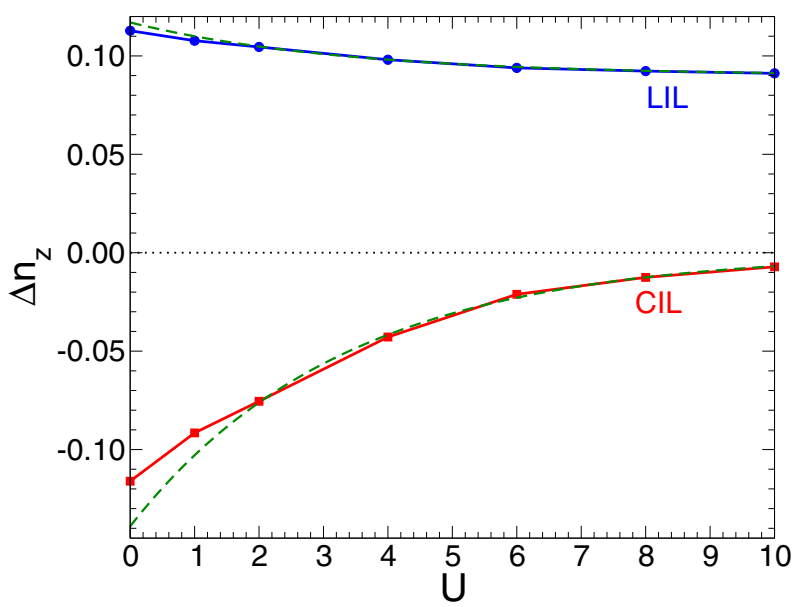

(c)

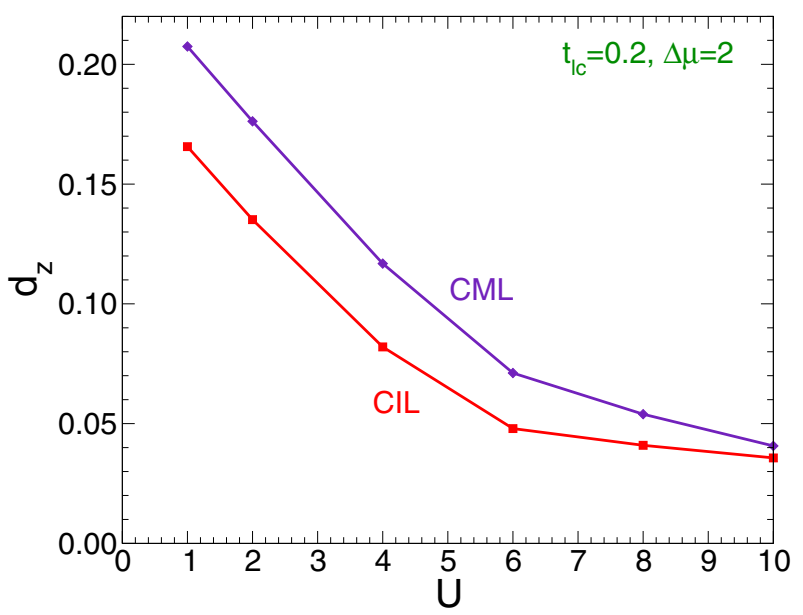

(e)

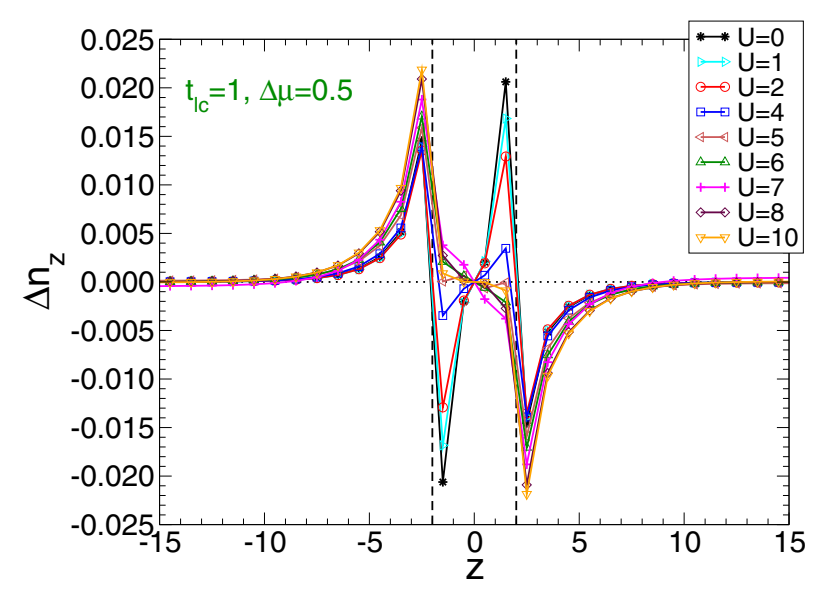

(b)

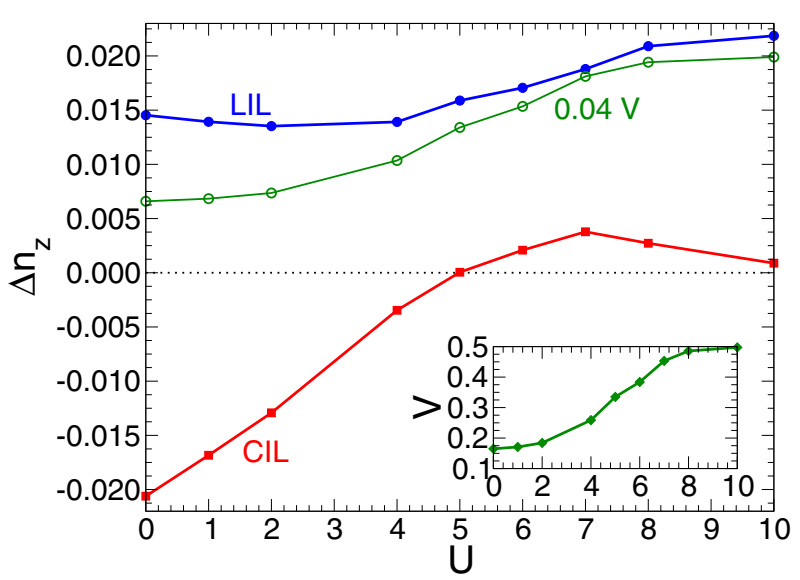

(d)

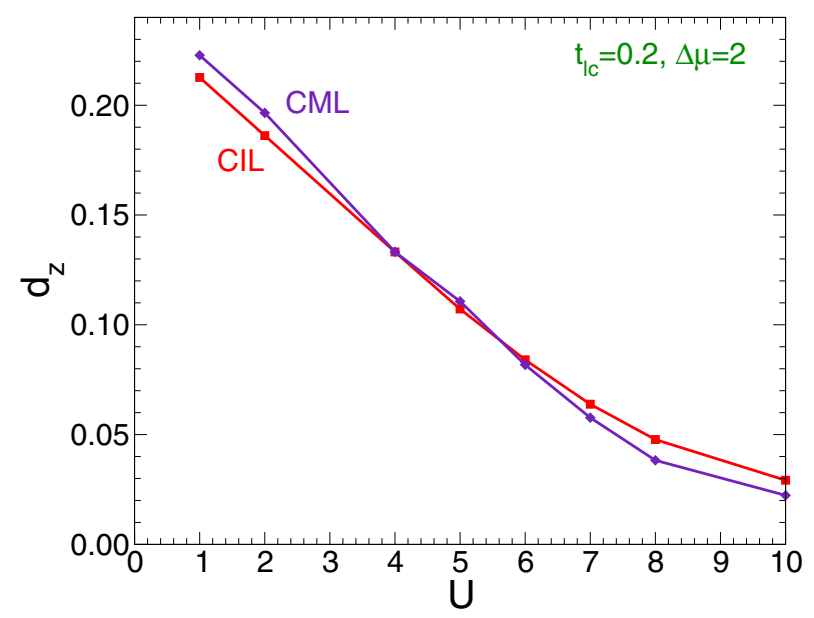

(f)

FIG. 5. $\Delta n_{z}$ (a), (b) as a function of layer index $z$ for different values of the Hubbard interaction $U$. (c), (d) $\Delta n_{z}$ for the LIL (blue curve) and for the CIL (red curve) as a function of Hubbard interaction $U$. Dashed green lines in (e) show results of the fit for the CIL $\Delta n_{\mathrm{CIL}}=A_{1} \exp \left(-A_{0} U\right)$ and for the LIL $\Delta n_{\mathrm{LIL}}=B_{2}+B_{1} \exp \left(-B_{0} U\right)$, with fit parameters $A_{0} \sim B_{0}=0.301, A_{1}=-0.139, B_{1}=0.027$, and $B_{2}=0.090$. In (d), we additionally plot the curves $0.04 * V$ versus $U$ (green). In the inset of (d), we plot the bias voltage as a function of interaction $U$ for fixed value of $\Delta \mu=0.5$ (see details in text). (e), (f) Double occupancy $d_{z}=\left\langle n_{z, \mathbf{r}, \uparrow} n_{z, \mathbf{r} \downarrow}\right\rangle$ for the CIL (red curve) and for the CML(indigo curve) as a function of Hubbard interaction $U$. The results in (a), (c), (e) are obtained with $\Delta \mu=2$ and $t_{\mathrm{lc}}=t_{\mathrm{rc}}=0.2$, while the ones in (b), (d), (f) with $\Delta \mu=0.5$ and $t_{\mathrm{lc}}=t_{\mathrm{rc}}=1$. Other parameters are the same as in Fig. 3. 
Remember that the bias voltage $V=v_{l}-v_{r}$ and the difference between the chemical potentials $\Delta \mu=\mu_{l}-\mu_{r}$ differ from each other. As we have already discussed in Sec. IID, it is numerically more convenient to perform calculations for fixed $\Delta \mu$ and evaluate $V$ a posteriori. For weak values of the LC-junction coupling or for large value of $U$, the difference between $V$ and $\Delta \mu$ is negligible ( $1 \%$ or smaller). However, there is a significant deviation for the case of $t_{\mathrm{lc}}=1$ and $U=4$, see Fig. 3(c). This is due the fact that when increasing $t_{\mathrm{lc}}$ the flow of particles from the left lead to the right one increases. As a result, there is a depletion of particles on the left lead which, if one wants to keep both leads at HF, has to be compensated by increasing $\mu_{l}$. The opposite situation obviously occurs on the right lead.

\section{B. Effect of the LC-junction coupling strength}

We further investigate the effect of the LC-junction coupling strength $t_{\mathrm{lc}}$ between the leads and the correlated region. We perform calculations for several values of $t_{\mathrm{lc}}$, fixing $\Delta \mu=2$ and again considering $U=4,8$.

When the LC-junction coupling strength is increased, the current through the heterostructure rises. Thus, we expect that more charge is transferred from the left lead to the correlated region. Indeed our results, Figs. 4(a) and 4(b), show that the charging of the LIL and the CIL are first decreasing as $t_{\mathrm{lc}}$ is increased. With further increase of $t_{\mathrm{lc}}$, this trend holds true for the LIL while, interestingly, for the CIL, $\Delta n_{\mathrm{CIL}}$ changes sign at some $U$-dependent value $t_{\mathrm{lc}}^{*}$. Furthermore, we find that $t_{\mathrm{lc}}^{*}$ decreases with increasing $U$ and for noninteracting correlated region $(U=0) \Delta n_{\mathrm{CIL}}$ is negative for all values of $t_{\mathrm{lc}}$ we have considered. From here, it follows then that correlations lead to an earlier disappearance of dipolelike layers with respect to the LC-junction coupling strength. This can be understood by the following.

For $U=0$, the behavior of the system can be intuitively understood by the hydraulic analogy, where a fluid takes over the role of the electric charge and pipes represent wires. In this picture, larger $t_{\mathrm{lc}}$ translates into a bigger diameter of the "LC-junction-pipe." For the behavior of the LIL, this means that less fluid gets jammed at the interface. When thinking about the behavior of the left-CIL in the hydraulic picture, it is

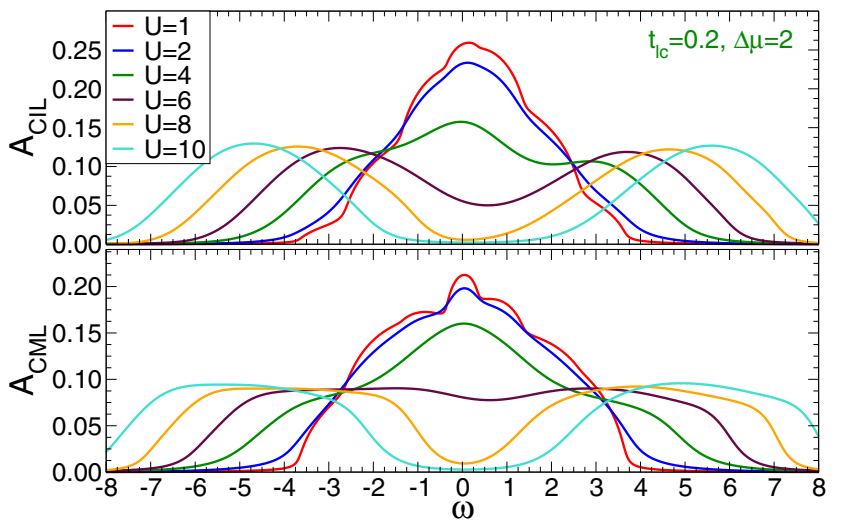

(a) easiest to consider the jam created at the right-CIL, since the two are connected by particle-hole symmetry, which will also get decreased with increasing $t_{\mathrm{lc}}$. This means that the trends observed in Fig. 4(b) are consistent with the hydraulic analogy.

Coming back to the reason why for stronger Hubbard interaction $t_{\mathrm{lc}}^{*}$ is lowered, we can thus interpret the slope of $\Delta n_{\mathrm{CIL}}\left(t_{\mathrm{lc}}\right)$, for low $t_{\mathrm{lc}}$, to originate from the $U=0$ behavior and thus the value of $t_{\mathrm{lc}}^{*}$ is mainly influenced by the starting value $\Delta n_{\mathrm{CIL}}\left(t_{\mathrm{lc}}=0\right)$, which is suppressed by the Hubbard interaction leading to the decrease of $t_{\mathrm{lc}}^{*}$ as a function of $U$.

\section{Effect of the local interaction}

Finally, we investigate the effect of the interaction $U$ for small $\left(t_{\mathrm{lc}}=0.2\right)$ and larger $\left(t_{\mathrm{lc}}=1\right)$ values of the LCjunction coupling strength. We consider differences between the chemical potentials, $\Delta \mu=2$ and $\Delta \mu=0.5$, respectively. These values are chosen such that for small interactions the opposite charging of the LIL and CIL is most pronounced, see Fig. 3(b). Furthermore, to better resolve the charge distribution, we also present results for a system with a larger correlated region $\left(L_{c}=40\right)$, in addition to the case with $L_{c}=4$. When studying the charging dependence as a function of $U$, we should expect that, in the limit of large $U, \Delta n_{z}$ vanishes for the correlated region, since in this limit any double occupation is extinguished.

\section{Small correlated region $\left(L_{c}=4\right)$}

First, we discus the effect of the interaction for weak LC-junction coupling $\left(t_{\mathrm{lc}}=0.2\right)$ and $\Delta \mu=2$. Figures 5(a) and 5(c) show that the opposite charging of the interface layers is suppressed by the Hubbard interaction. Further, $\Delta n_{z}$ for LIL converges monotonically to some finite value for $U \rightarrow \infty$, while for the CIL it converges to 0 as expected. To investigate the behavior of the boundary charge, we fit them (for $U \geqslant 2$ ) with exponential functions [see Fig. 5(c)], namely $\Delta n_{\mathrm{CIL}}=A_{1} \exp \left(-A_{0} U\right)$ and $\Delta n_{\mathrm{LIL}}=B_{2}+B_{1} \exp \left(-B_{0} U\right)$. The resulting fit parameters are given in the figure caption. Notice that both fits give approximately the same exponent; that is, $A_{0} \approx B_{0}$.

For small LC-junction coupling $\left(t_{\mathrm{lc}}=0.2\right)$ and increasing interaction strength $U$, as we already mentioned above, the

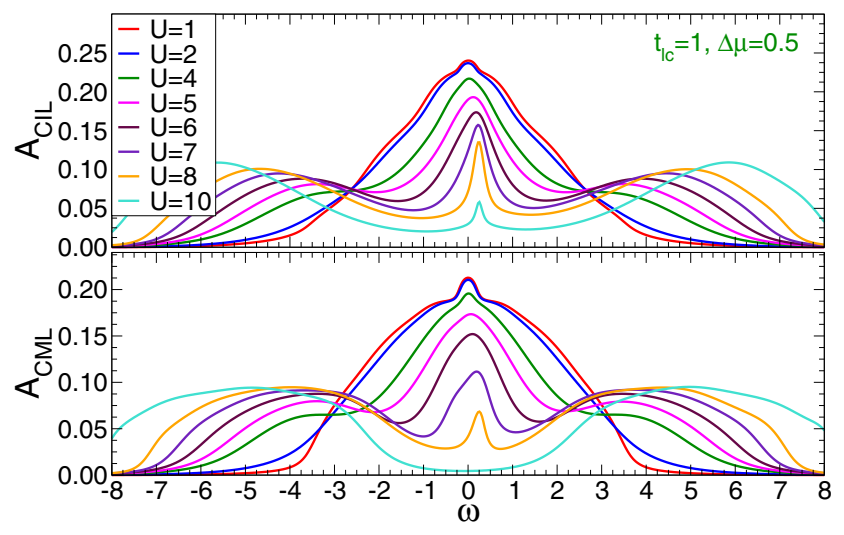

(b)

FIG. 6. Steady-state spectral function for different Hubbard interactions for the CIL, upper panel, and central middle layer (CML), lower panel. Same setup as in Fig. 5. (a) $\Delta \mu=2$ and LC-junction coupling $t_{\mathrm{lc}}=t_{\mathrm{rc}}=0.2$, (b) $\Delta \mu=0.5$ and LC-junction coupling $t_{\mathrm{lc}}=t_{\mathrm{rc}}=1$. 
charging of the LIL and CIL is exponentially suppressed, but these layers still have opposite signs and for any finite $U$, while being reduced, the dipolelike layers are still there.

On the other hand, this is no longer the case for stronger LC-junction coupling strength $\left(t_{\mathrm{lc}}=1\right)$ and $\Delta \mu=0.5$, as can be anticipated based on the results presented in previous subsections. Indeed, from Figs. 5(b) and 5(d), we can see that $\Delta n_{z}$ is nonmonotonic for both surface layers and, in addition, the CIL displays a sign change at $U \approx 5$ which approaches zero only for higher values of the interaction.

To understand this behavior, it is important to recall that the results presented in Figs. 5(b) and 5(d) are performed for fixed $\Delta \mu=0.5$, which corresponds to different bias voltages $V$ [see inset of Fig. 5(d)]. When examining Fig. 5(d) more closely, one can see that the shape of $\Delta n_{\mathrm{LIL}}(U)$ for $U>4$ resembles that of $V(U)$ from the inset. Moreover, from Fig. 3(b), we know that $\Delta n_{\mathrm{LIL}}(V)$ is just proportional to $V$ and almost insensitive to $U$. Based on that, to exclude the dependence on the bias voltage we plot $n(U)=0.04 \mathrm{~V}(U)$, where the coefficient of proportionality is extracted from Fig. 3(b), see green line in Fig. 5(d). One indeed finds that the behavior of $\Delta n_{\mathrm{LIL}}$ for $U>4$ is controlled by the $V(U)$ dependency. We thus expect that the curve of $\Delta n_{\mathrm{LIL}}$ vs $U$ for fixed $V$ would continue its downward trend also for $U>4$ and converge to some value as in the case of the smaller LC-junction coupling strength $t_{\mathrm{lc}}=0.2$. In contrast to the behavior of $\Delta n_{\mathrm{LIL}}$, fixing $V$ would not affect qualitatively the behavior of $\Delta n_{\mathrm{CIL}}$ versus $U$. As a matter of fact, taking the dependence on $V$ into account, one would expect an even more pronounced maximum in the behavior of $\Delta n_{\mathrm{CIL}}$ [see red curve in Fig. 5(d)].

We also investigate the double occupancy $d_{z}=$ $\left\langle n_{z, \mathbf{r}, \uparrow} n_{z, \mathbf{r} \downarrow}\right\rangle$. Our calculations show that both for small as well as for large LC-junction coupling strength, the double occupancies $d_{z}$ for the correlated sites are monotonically decreasing as expected [see Figs. 5(e) and 5(f)]. For weak LC-junction coupling strength, the double occupancy $d_{z}$ of the CIL is always larger compared to the one of the correlated middle layer (CML), while for large LC-junction coupling strength, this is only true for $U \lesssim 5$. This can be explained by the fact that for $U \lesssim 5$, the filling in the CML is larger than the filling in the CIL.

A different behavior of the system between the regimes of weak and strong LC-junction coupling strengths can be also seen by considering the steady-state spectral functions $A_{z}(\omega)=-\frac{1}{\pi} \Im m G_{z}^{R}(\omega)$ (see Fig. 6). For $t_{\mathrm{lc}}=0.2$ and $\Delta \mu=$ 2 , the spectral function does not show a Kondo-like peak at $\omega=\mu_{l}=1$. We attribute this fact to a combined effect of the width of the Kondo-like peak being so small that we are not able to resolve it as well as the substantial bias voltage present in the system, leading to decoherence which suppresses the resonance. In contrast, for large values of the LC-junction coupling strength there is a clear Kondo-like peak for the CIL (at $\omega=\mu_{l}=0.25$ ) up to interactions as strong as $U=10$. This is not surprising, because the width of the Kondo-like peak is proportional to $t_{\mathrm{lc}}^{2}$ and, correspondingly, the difference between these two cases is $O(100)$ and, in addition, the considered $\Delta \mu$ is a factor of 4 smaller. Figure 6 also shows the spectral function for the CML featuring, as expected [86] due to the increased distance to the leads, a less pronounced Kondo-like

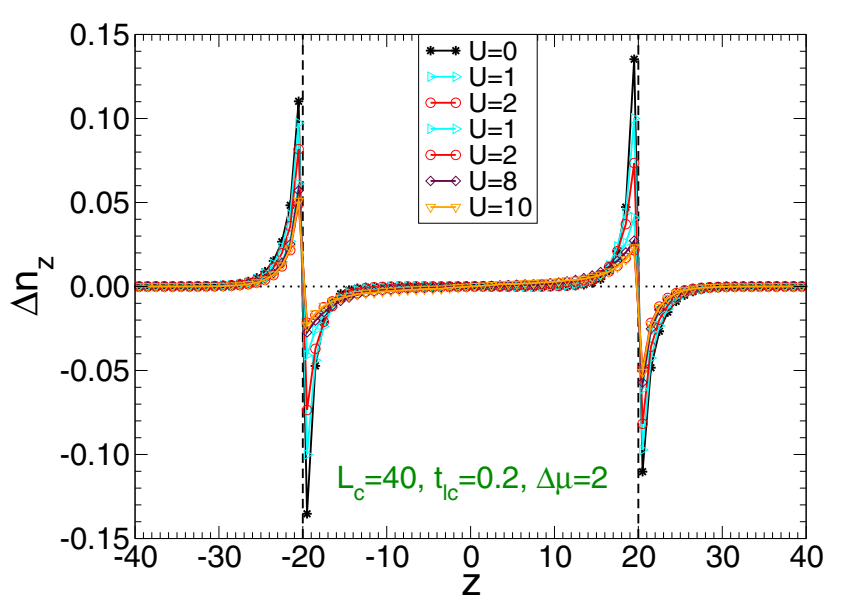

(a)

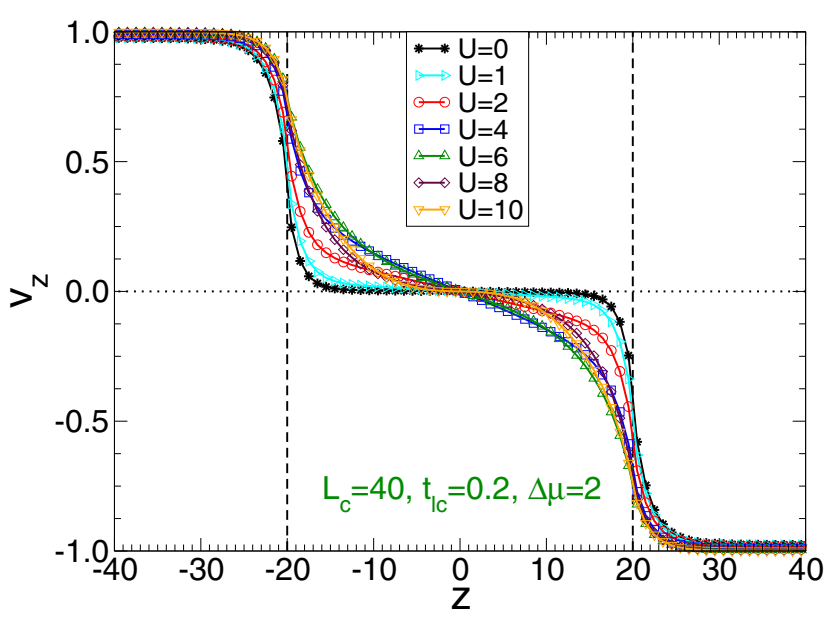

(b)

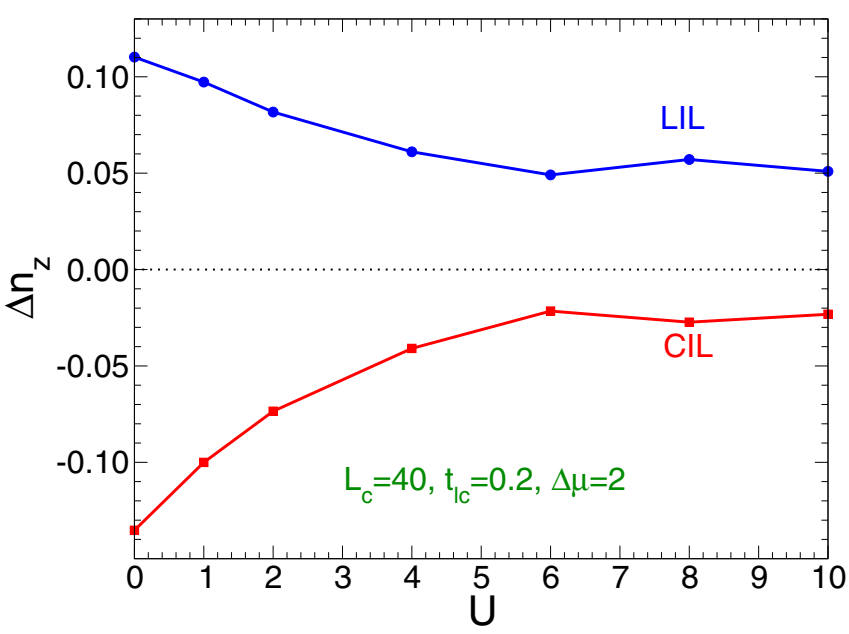

(c)

FIG. 7. $\Delta n_{z}$ (a) and on-site energies $v_{z}$ (b) as a function of layer index $z$ for $L_{c}=40$ correlated layers, $t_{\mathrm{lc}}=t_{\mathrm{rc}}=0.2, \Delta \mu=2$, and different values of Hubbard interaction $U$. Total number of layers $L=100$. Calculations are performed with $N_{b}=4$. Other parameters are the same as in Fig. 3. (c) $\Delta n_{z}$ for the LIL (blue curve) and CIL (red curve) as a function of the Hubbard interaction $U$. 
peak compared to the CIL which is already destroyed for $U=10$.

It appears that the Kondo-like peak in the spectral density occurs whenever $\Delta n_{\text {LIL }}$ and $\Delta n_{\text {CIL }}$ have the same sign, which indicates that the mobility within the correlated region is small as compared to $t_{\mathrm{lc}}$.

\section{Large correlated region $\left(L_{c}=40\right)$}

We now want to investigate how far the charging of the interface region extends into a bulk system. To this end, we enlarge the correlated region to $L_{c}=40$. Results are obtained with $N_{b}=4$ auxiliary bath sites in the AMEA impurity solver [85]. Due to the heavy numerical calculations, the convergence of the DMFT self-consistency is quite slow, especially for the strong interactions.

At this point it is worth noting that for a metallic material, one would expect that only the surface is charged with an exponential tail into the bulk since the induced charge on the surface will compensate the electric field in the bulk. Indeed, our results for $\Delta n_{z}$ and $v_{z}$, presented in Figs. 7(a) and 7(b), respectively, show that the charging and on-site energies behave as expected and fall off exponentially into the bulk. Further, we find that the corresponding penetration depth for charging, although increasing with $U$, depends only weakly on $U$ and that this dependence is more pronounced for the on-site energies. Note that the system is still metallic for all values of the interaction $U \leqslant 10$ and the exponential suppression can therefore be attributed to screening. The trend that the penetration depth increases with $U$ can thus be interpreted as less effective screening due to the lower DOS around $\omega \approx 0$.

As in the previous results for $L_{c}=4$, the main effect of the interaction is to reduce the absolute value of the charging at the interface between the correlated and uncorrelated region. As can be seen from Fig. 7(c), the behavior agrees qualitatively with the ones observed for $L_{c}=4$, see also Fig. 5(c). The fact that the exponential dependence on $U$ is not so obvious in Fig. 7(c) can be attributed to the lower accuracy due to the increased numerical challenge to converge the self-consistent equations.

\section{Current}

We also investigate the effect of the interaction on the steady-state current density through the correlated interface. The latter can be calculated using off-diagonal elements of the Keldysh Green's function $[12,87]$ :

$$
J=J_{z, z+1}=t_{z, z+1} \int_{-\infty}^{\infty} \frac{d \omega}{2 \pi} \int_{\mathrm{BZ}} \frac{d^{2} \mathbf{k}}{(2 \pi)^{2}}\left(\mathbf{G}_{z+1, z}^{K}-\mathbf{G}_{z, z+1}^{K}\right),
$$

where summation over spin is implicitly assumed.

Results are shown in Fig. 8, where we plot a rescaled current density $J / t_{\mathrm{lc}}$ to present the curves on the same plot. As expected, our calculations show that for all considered system parameters the current density is strongly suppressed when increasing the interaction strength $U$ [88]. For the system with a smaller correlated region $\left(L_{c}=4\right)$, the qualitative form of the suppression as a function of $U$ seems rather independent of $t_{\mathrm{lc}}$ and $\Delta \mu$. Nevertheless, from the figure it appears that the

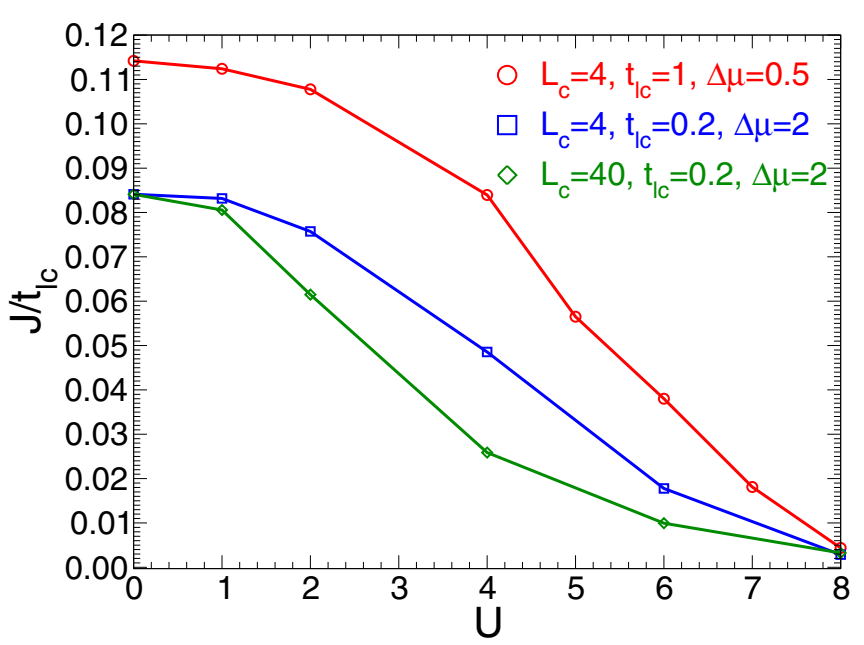

FIG. 8. Current density $J$ as a function of the interaction for different parameter sets. Blue line with squares (red line with circles) correspond to a system with $L_{c}=4$ correlated layers, with LCjunction coupling strength $t_{\mathrm{lc}}=0.2\left(t_{\mathrm{lc}}=1\right)$ and $\Delta \mu=2(\Delta \mu=$ 0.5 ). Green diamonds correspond to a system with $L_{c}=40$ correlated sites with $t_{\mathrm{lc}}=0.2$ and $\Delta \mu=2$. Other parameters are the same as in Fig. 3.

scaling behavior of the current density is stronger than $\propto t_{\mathrm{lc}}$. This is because the stronger hybridization leads to a more pronounced resonance peak making the central region more metallic especially around $\omega=0$ resulting in more spectral weight within the Fermi window of the leads already for small voltages. See also Figs. 6(a) and 6(b).

Furthermore, we compare the steady-state current density for the small $\left(L_{c}=4\right)$ and the large $\left(L_{c}=40\right)$ correlated regions (see Fig. 8). We observe that the difference between them is marginal for weak interactions while for intermediate to strong interactions we have a substantial suppression for $L_{c}=40$. This is due to a reduced electron mobility induced by the loss of metallicity of the correlated region. However, this cannot be simply generically described by a decreased conductivity but rather by the fact that for $U \gtrsim 2$, the penetration depth of the electric field exceeds the size of the small correlated region $L_{c}=4$, see also Fig. 7(b).

\section{CONCLUSIONS}

We addressed the steady-state properties of a system consisting of a multilayer correlated region attached to two metallic leads. The model was solved by nonequilibrium R-DMFT whereby AMEA $[62,63,71]$ was used as the impurity solver. We studied the charge redistribution in the system induced by the local Hubbard and the LRCIs in the presence of a bias voltage. We find that its behavior is very different for weak and strong LC-junction coupling strengths, especially for strong local interactions. The influence of $U$ on the lead layers is due to the proximity effect and therefore less pronounced in the lead compared to the correlated region.

Our results indicate that the charges (considered with respect to the bulk value) on opposite sides of the LC-junction can have equal or opposite signs, depending on the system parameters. The case of opposite signs can be interpreted as the 


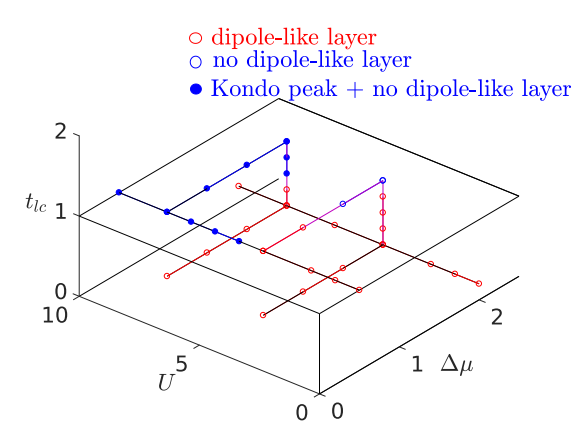

FIG. 9. Three-dimensional representation of the regions in $t_{\mathrm{lc}}-$ $\Delta \mu-U$-space in which the system exhibits(lacks) dipolelike layers at the LC-junction red (blue) open circles. In addition, parameter combinations where a Kondo-like peak can be clearly identified, are marked by full circles.

formation of a dipolelike layer. In particular, these dipolelike layers are present for small but finite LC-junction coupling strengths. In contrast, for stronger values of the LC-junction coupling strength, this is only true for intermediate to weak interactions at low bias voltages. For strong interactions, as well as for intermediate to weak interactions at moderate to high values of the bias voltage, the dipolelike layers are destroyed and the charging of the LIL and CIL have the same sign. The dependence of $\Delta n_{\mathrm{CIL}}$ on the local Hubbard interaction $U$ is quite peculiar, being exponentially decreasing for small $t_{\mathrm{lc}}$ while for large $t_{\mathrm{lc}}$ it displays a nonmonotonic behavior and even changes sign as a function of $U$.

This behavior can be understood from the fact that the dipolelike layers are formed if the charges flow faster out of the transition region than they flow in, i.e.,

$$
\frac{t_{c, \text { eff }}}{t_{l c}}>1,
$$

where $t_{c, \text { eff }}$ is the effective hopping for the correlated region. Indeed, we observe that for sufficiently large $t_{\mathrm{lc}}$, the dipolelike layers get destroyed in accordance with Eq. (23). Obviously, increasing the Hubbard interaction effectively decreases the mobility in the correlated region. We also observe that a Kondolike peak is present in the spectral function of the CIL for large values of the LC-junction coupling strength $t_{\mathrm{lc}}$ and the Hubbard interaction $U$. This suggests that the dipolelike layers have the tendency to suppress the Kondo-like peak. A summary of our results is reported in the three-dimensional plot of Fig. 9.

Finally, we want to emphasize that the results presented in this paper obtained for the Hubbard interaction differ from the ones for the Falicov-Kimball model for large values of the LC-junction coupling $\left(t_{\mathrm{lc}}=1\right)$ in Ref. [20]. In the latter, the LIL and CIL are always oppositely charged. This indicates that the sign change of $\Delta n_{\mathrm{CIL}}$ is not a generic feature of strong local correlations paired with long-range coulomb forces. Rather it is a combined effect of strong local Hubbard interactions together with long-range Coulomb forces.

\section{ACKNOWLEDGMENTS}

We thank Walter Hofstetter and Martin Eckstein for valuable discussions. This work was supported by the Austrian Science Fund (FWF): P26508, as well as SfB-ViCoM Project
No. F04103, and NaWi Graz. The calculations were partly performed on the D-Cluster Graz and on the VSC-3 cluster Vienna.

\section{APPENDIX: POISSON EQUATION}

Here, we present the details of the self-consistent solution of Eqs. (11)-(13). As mentioned in the main text, we employ the Newton-Raphson method to find the root of

$$
\Phi_{z}(\vec{v})=c_{z}\left[\frac{\partial}{\partial z}\left(\frac{1}{c_{z}} \frac{\partial v_{z}}{\partial z}\right)+\left(n_{z}-n^{\text {bulk }}\right)\right]
$$

First, we discretize the derivative. Setting the lattice constant $a=1$, we get

$$
\begin{aligned}
\Phi_{z}(\vec{v})= & v_{z+1}-2 v_{z}+v_{z-1}+\frac{\varepsilon_{r, z+1}-\varepsilon_{r, z-1}}{2 \varepsilon_{r, z}} \frac{v_{z+1}-v_{z-1}}{2} \\
& +\frac{1}{\varepsilon_{r, z} \varepsilon_{0}}\left(n_{z}-n^{\text {bulk }}\right) .
\end{aligned}
$$

Following Newton-Raphson, we expand

$$
\Phi_{j}(\vec{v}+\Delta \vec{v})=\Phi_{j}(\vec{v})+\sum_{i} \frac{\partial \Phi_{j}(\vec{v})}{\partial v_{i}} \Delta v_{i}
$$

Here $\Delta \vec{v}=\vec{v}^{(n+1)}-\vec{v}^{(n)}$ is the difference between two consecutive iterations in the Poisson loop. Assuming $\Phi_{z}(\vec{v}+\Delta \vec{v}) \stackrel{!}{=}$ 0 , we obtain

$$
\Phi_{j}(\vec{v})=-M_{j i}\left(v_{i}^{(n+1)}-v_{i}^{(n)}\right),
$$

with

$$
M_{j i}=\frac{\partial}{\partial v_{i}} \Phi_{j}(\vec{v}),
$$

which leads to the final iteration scheme

$$
\vec{v}^{(n+1)}=\vec{v}^{(n)}-M^{-1} \boldsymbol{\Phi}(\vec{v}) .
$$

\section{Expressions for the matrix Elements $M_{j i}$}

Plugging Eq. (A1) into Eq. (A5), we obtain

$$
M_{j i}=\underbrace{\frac{\partial}{\partial v_{i}}\left[\frac{1}{\varepsilon_{r, j}} \frac{\partial}{\partial z}\left(\varepsilon_{r, j} \frac{\partial v_{j}}{\partial z}\right)\right]}_{\equiv M_{j i}^{(1)}}+\underbrace{\frac{\partial}{\partial v_{i}}\left[\frac{1}{\varepsilon_{r, j} \varepsilon_{0}} n_{j}(\vec{v})\right]}_{M_{j i}^{(2)}} .
$$

Here, we used $c_{z} \equiv \frac{1}{\varepsilon_{0} \varepsilon_{r} z}$ and the fact that $n^{\text {bulk }}$ does not depend on $\vec{v}$ and, therefore, $\partial n^{\text {bulk }} / \partial v_{i}=0$. 
After some simple manipulations, we arrive at

$$
\begin{aligned}
M_{j i}^{(1)} & \equiv \frac{\partial}{\partial v_{i}}\left[\frac{1}{\varepsilon_{r, j}} \frac{\partial}{\partial z}\left(\varepsilon_{r, j} \frac{\partial v_{j}}{\partial z}\right)\right] \\
& =\left(1-\frac{\delta \varepsilon_{r, j}}{4 \varepsilon_{r, j}}\right) \delta_{i, j-1}-2 \delta_{i, j}+\left(1+\frac{\delta \varepsilon_{r, j}}{4 \varepsilon_{r, j}}\right) \delta_{i, j+1},
\end{aligned}
$$

with $\delta \varepsilon_{r, j}=\varepsilon_{r, j+1}-\varepsilon_{r, j-1}$.

This leaves us with the evaluation of the matrix elements for $M^{(2)}$, which involves the dependence of the charge density on the on-site energies. Using, the defining equations, Eqs. (10) and (13) in Eq. (A7), we obtain

$$
\begin{aligned}
M_{j i}^{(2)} & =\frac{e^{2}}{\varepsilon_{r, j} \varepsilon_{0}} \frac{\partial}{\partial v_{i}} n_{j} \\
& =\frac{e^{2}}{\varepsilon_{r, j} \varepsilon_{0}} \int_{\mathrm{BZ}} \frac{d^{2} \boldsymbol{k}}{(2 \pi)^{2}} \int \frac{d \omega}{2 \pi} \Im m\left(\frac{\partial}{\partial v_{i}} G_{j j}^{K}(\omega, \boldsymbol{k})\right) .
\end{aligned}
$$

Next, using the Keldysh inversion formula, $G^{K}=$ $-G^{R}\left[G^{-1}\right]^{K} G^{A}$, we can expand the derivative

$$
\begin{aligned}
\frac{\partial}{\partial v_{i}} G_{j j}^{K}= & -\frac{\partial G_{j l}^{R}}{\partial v_{i}}\left[G^{-1}\right]_{l l^{\prime}}^{K} G_{l^{\prime} j}^{A}-G_{j l}^{R}\left[G^{-1}\right]_{l l^{\prime}}^{K} \frac{\partial G_{l^{\prime} j}^{A}}{\partial v_{i}} \\
& -G_{j l}^{R} \frac{\partial}{\partial v_{i}}\left[G^{-1}\right]_{l l^{\prime}}^{K} G_{l^{\prime} j}^{A} .
\end{aligned}
$$

Here and below, all indices appearing twice are summed over. Relating the derivative $\frac{\partial G_{j l}^{\gamma=R, A}}{\partial v_{i}}$ to the derivative of its inverse, given by Eq. (3) [89], leads to

$$
\begin{aligned}
\frac{\partial G_{j l}^{\gamma=R, A}}{\partial v_{i}} & =-G_{j l^{\prime}}^{\gamma} \frac{\partial}{\partial v_{i}}\left[G^{\gamma}\right]_{l^{\prime} l^{\prime \prime}}^{-1} G_{l^{\prime \prime} l}^{\gamma} \\
& =G_{j i}^{\gamma} G_{i l}^{\gamma}+G_{j i}^{\gamma} G_{i l}^{\gamma} \frac{\partial}{\partial v_{i}} \Sigma_{\mathrm{hyb}, i}^{\gamma},
\end{aligned}
$$

and recalling Eq. (4), we also have

$$
\frac{\partial}{\partial v_{i}}\left[G^{-1}\right]_{l l^{\prime}}^{K}=-\delta_{l i} \delta_{l^{\prime} i} \frac{\partial}{\partial v_{i}} \Sigma_{\mathrm{hyb}, i}^{\gamma} .
$$

Thus, Eq. (A10) now reads

$$
\begin{aligned}
\frac{\partial}{\partial v_{i}} G_{j j}^{K}= & G_{j i}^{R} G_{i j}^{K}+G_{j i}^{K} G_{i j}^{A}+G_{j i}^{R} G_{i j}^{K} \frac{\partial}{\partial v_{i}} \Sigma_{\mathrm{hyb}, i}^{R} \\
& +G_{j i}^{K} G_{i j}^{A} \frac{\partial}{\partial v_{i}} \Sigma_{\mathrm{hyb}, i}^{A}+G_{j i}^{R} G_{i j}^{A} \frac{\partial}{\partial v_{i}} \Sigma_{\mathrm{hyb}, i}^{K},
\end{aligned}
$$

which, based on the symmetries of the Green's function and the fluctuation dissipation theorem for $\Sigma_{\mathrm{hyb}, i}^{K}$ allows the simplification to the final form

$$
\frac{\partial}{\partial v_{i}} G_{j j}^{K}=2 i \Im m\left[G_{j i}^{R} G_{i j}^{K}\right]+N_{j \kappa}^{b} \delta_{\kappa 1}+N_{j \kappa}^{b} \delta_{\kappa L} .
$$

Here

$$
N_{j \kappa}^{b}=2 i \Im m\left[\eta_{\kappa}\left(G_{j \kappa}^{R} G_{\kappa j}^{K}+\left|G_{j \kappa}^{R}\right|^{2}\left(1-2 f_{\kappa}\right)\right)\right]
$$

and

$$
\eta_{\kappa}=-\frac{1}{2}\left(1+i \frac{\omega-v_{\kappa}-v_{\kappa}^{(0)}-E(\boldsymbol{k})}{\sqrt{4 t_{\kappa}^{2}-\left(\omega-v_{\kappa}-v_{\kappa}^{(0)}-E(\boldsymbol{k})\right)^{2}}}\right) .
$$

Moreover $f_{\kappa=1, L}$ stands for the Fermi function in left and right leads, respectively.

To speed up the convergence, we can use the fact that the electron density in the first and last site will converge to their bulk values and therefore we consider them fixed, which also means $\partial n_{j=1, l} / \partial v_{i}=0$ and, correspondingly, $M_{j i}^{(2)}=0$ for $j \in\{1, L\}$.
[1] M. Izumi, Y. Ogimoto, Y. Konishi, T. Manako, M. Kawasaki, and Y. Tokura, Mater. Sci. Eng. B 84, 53 (2001).

[2] A. Ohtomo, D. A. Muller, J. L. Grazul, and H. Y. Hwang, Nature 419, 378 (2002).

[3] S. Gariglio, C. H. Ahn, D. Matthey, and J.-M. Triscone, Phys. Rev. Lett. 88, 067002 (2002).

[4] C. H. Ahn, S. Gariglio, P. Paruch, T. Tybell, L. Antognazza, and J.-M. Triscone, Science 284, 1152 (1999).

[5] A. Ohtomo and H. Y. Hwang, Nature 427, 423 (2004).

[6] Q. X. Zhu, W. Wang, X. Q. Zhao, X. M. Li, Y. Wang, H. S. Luo, H. L. W. Chan, and R. K. Zheng, J. Appl. Phys. 111, 103702 (2012).

[7] H. Zenia, J. K. Freericks, H. R. Krishnamurthy, and T. Pruschke, Phys. Rev. Lett. 103, 116402 (2009).

[8] S. T. F. Hale and J. K. Freericks, Phys. Rev. B 83, 035102 (2011).

[9] M. Knap, W. von der Linden, and E. Arrigoni, Phys. Rev. B 84, 115145 (2011).

[10] G. Mazza, A. Amaricci, M. Capone, and M. Fabrizio, Phys. Rev. B 91, 195124 (2015).
[11] P. Ribeiro, A. E. Antipov, and A. N. Rubtsov, Phys. Rev. B 93, 144305 (2016).

[12] S. Okamoto, Phys. Rev. B 76, 035105 (2007).

[13] S. Okamoto, Phys. Rev. Lett. 101, 116807 (2008).

[14] M. Eckstein and P. Werner, Phys. Rev. Lett. 113, 076405 (2014).

[15] M. E. Sorantin, A. Dorda, K. Held, and E. Arrigoni, Phys. Rev. B 97, 115113 (2018).

[16] M. Eckstein and P. Werner, Phys. Rev. B 88, 075135 (2013).

[17] I. Titvinidze, A. Dorda, W. von der Linden, and E. Arrigoni, Phys. Rev. B 94, 245142 (2016).

[18] K. Steffen, R. Frésard, and T. Kopp, Phys. Rev. B 95, 035143 (2017).

[19] P. Bakalov, B. Ydens, and J. Locquet, Phys. Status Solidi A 211, 440 (2014).

[20] S. T. F. Hale and J. K. Freericks, Phys. Rev. B 85, 205444 (2012).

[21] J. K. Freericks and V. Zlatić, Phys. Status Solidi B 244, 2351 (2007).

[22] P. Bakalov, D. Nasr Esfahani, L. Covaci, F. M. Peeters, J. Tempere, and J.-P. Locquet, Phys. Rev. B 93, 165112 (2016). 
[23] A. Georges, G. Kotliar, W. Krauth, and M. J. Rozenberg, Rev. Mod. Phys. 68, 13 (1996).

[24] D. Vollhardt, in Lecture Notes on the Physics of Strongly Correlated Systems, AIP Conf. Proc. No. 1297, edited by A. Avella and F. Mancini (AIP, New York, 2010), pp. 339-403.

[25] W. Metzner and D. Vollhardt, Phys. Rev. Lett. 62, 324 (1989).

[26] M. Potthoff and W. Nolting, Phys. Rev. B 59, 2549 (1999).

[27] M. Potthoff and W. Nolting, Phys. Rev. B 60, 7834 (1999).

[28] M. Potthoff and W. Nolting, Eur. Phys. J. B 8, 555 (1999).

[29] M. Potthoff and W. Nolting, Phys. B: Condens. Matter 259-261, 760 (1999).

[30] J. K. Freericks, Transport in Multilayered Nanostructures (Imperial College Press, London, 2006).

[31] R. Nourafkan, F. Marsiglio, and M. Capone, Phys. Rev. B 82, 115127 (2010).

[32] H. Ishida and A. Liebsch, Phys. Rev. B 79, 045130 (2009).

[33] R. Nourafkan and F. Marsiglio, Phys. Rev. B 83, 155116 (2011).

[34] S. Okamoto, Phys. Rev. B 84, 201305 (2011).

[35] P. Miller and J. K. Freericks, J. Phys.: Condens. Matter 13, 3187 (2001).

[36] J. K. Freericks, Phys. Rev. B 70, 195342 (2004).

[37] S. Okamoto and A. J. Millis, Phys. Rev. B 70, 241104 (2004).

[38] V. Dobrosavljević and G. Kotliar, Phys. Rev. Lett. 78, 3943 (1997).

[39] V. Dobrosavljević and G. Kotliar, Philos. Trans. R. Soc., A 356, 57 (1998).

[40] Y. Song, R. Wortis, and W. A. Atkinson, Phys. Rev. B 77, 054202 (2008).

[41] J. Wernsdorfer, G. Harder, U. Schollwoeck, and W. Hofstetter, arXiv:1108.6057.

[42] R. W. Helmes, T. A. Costi, and A. Rosch, Phys. Rev. Lett. 100, 056403 (2008).

[43] A. Koga, T. Higashiyama, K. Inaba, S. Suga, and N. Kawakami, J. Phys. Soc. Jpn. 77, 073602 (2008).

[44] A. Koga, T. Higashiyama, K. Inaba, S. Suga, and N. Kawakami, Phys. Rev. A 79, 013607 (2009).

[45] K. Noda, A. Koga, N. Kawakami, and T. Pruschke, Phys. Rev. A 80, 063622 (2009).

[46] A. Koga, J. Bauer, P. Werner, and T. Pruschke, Phys. E: LowDimensional Syst. Nanostruct. 43, 697 (2011), Proceedings of the International Symposium on Nanoscience and Quantum Physics (nanoPHYS '09).

[47] N. Blümer and E. Gorelik, Comput. Phys. Commun. 182, 115 (2011), Computer Physics Communications Special Edition for Conference on Computational Physics Kaohsiung, Taiwan, Dec. 15-19, 2009 .

[48] D.-H. Kim, J. J. Kinnunen, J.-P. Martikainen, and P. Törmä, Phys. Rev. Lett. 106, 095301 (2011).

[49] M. W. Aulbach, F. F. Assaad, and M. Potthoff, Phys. Rev. B 92, 235131 (2015).

[50] M. Snoek, I. Titvinidze, C. Take, K. Byczuk, and W. Hofstetter, New J. Phys. 10, 093008 (2008).

[51] M. Snoek, I. Titvinidze, and W. Hofstetter, Phys. Rev. B 83, 054419 (2011).

[52] I. Titvinidze, A. Schwabe, N. Rother, and M. Potthoff, Phys. Rev. B 86, 075141 (2012).

[53] E. V. Gorelik, I. Titvinidze, W. Hofstetter, M. Snoek, and N. Blümer, Phys. Rev. Lett. 105, 065301 (2010).
[54] A. Schwabe, I. Titvinidze, and M. Potthoff, Phys. Rev. B 88, 121107 (2013).

[55] M. W. Aulbach, I. Titvinidze, and M. Potthoff, Phys. Rev. B 91, 174420 (2015).

[56] H. Aoki, N. Tsuji, M. Eckstein, M. Kollar, T. Oka, and P. Werner, Rev. Mod. Phys. 86, 779 (2014).

[57] P. Schmidt and H. Monien, arXiv:cond-mat/0202046.

[58] J. K. Freericks, V. M. Turkowski, and V. Zlatić, Phys. Rev. Lett. 97, 266408 (2006).

[59] J. K. Freericks, Phys. Rev. B 77, 075109 (2008).

[60] A. V. Joura, J. K. Freericks, and T. Pruschke, Phys. Rev. Lett. 101, 196401 (2008).

[61] M. Eckstein, M. Kollar, and P. Werner, Phys. Rev. Lett. 103, 056403 (2009).

[62] E. Arrigoni, M. Knap, and W. von der Linden, Phys. Rev. Lett. 110, 086403 (2013)

[63] I. Titvinidze, A. Dorda, W. von der Linden, and E. Arrigoni, Phys. Rev. B 92, 245125 (2015).

[64] A. Dorda, I. Titvinidze, and E. Arrigoni, J. Phys.: Conf. Ser. 696, 012003 (2016).

[65] R. Kubo, J. Phys. Soc. Jpn. 12, 570 (1957).

[66] J. Schwinger, J. Math. Phys. 2, 407 (1961).

[67] G. Baym and L. P. Kadanoff, Phys. Rev. 124, 287 (1961).

[68] L. P. Kadanoff and G. Baym, Quantum Statistical Mechanics: Green's Function Methods in Equilibrium and Nonequilibrium Problems (Addison-Wesley, Redwood City, CA, 1962).

[69] L. V. Keldysh, Zh. Eksp. Teor. Fiz. 47, 1515 (1965) [JETP 20, 1018 (1965)].

[70] P. W. Anderson, Phys. Rev. 124, 41 (1961).

[71] A. Dorda, M. Nuss, W. von der Linden, and E. Arrigoni, Phys. Rev. B 89, 165105 (2014).

[72] W. POTZ, J. Appl. Phys. 66, 2458 (1989).

[73] H. Haug and A.-P. Jauho, Quantum Kinetics in Transport and Optics of Semiconductors (Springer, Heidelberg, 1998).

[74] J. Rammer and H. Smith, Rev. Mod. Phys. 58, 323 (1986).

[75] A. Dorda, M. Sorantin, W. von der Linden, and E. Arrigoni, New J. Phys. 19, 063005 (2017).

[76] R. Haydock, Solid State Physics, Advances in Research and Applications, edited by H. Ehrenreich, F. Seitz, and D. Turnbull, Vol. 35 (Academic, London, Academic, 1980).

[77] D. J. Thouless and S. Kirkpatrick, J. Phys. C 14, 235 (1981).

[78] C. H. Lewenkopf and E. R. Mucciolo, J. Comput. Electron. 12, 203 (2013).

[79] A. Dorda, M. Ganahl, H. G. Evertz, W. von der Linden, and E. Arrigoni, Phys. Rev. B 92, 125145 (2015).

[80] H.-P. Breuer, B. Kappler, and F. Petruccione, Phys. Rev. A 56, 2334 (1997).

[81] M. Sorantin, D. Fugger, A. Dorda, W. von der Linden, and E. Arrigoni (unpublished).

[82] Our formalism can be easily generalized in the case when filling of the layers are different from each other. In this case, we should just change $n^{\text {bulk }}$ by $n_{z}^{\text {bulk }}$.

[83] Recall that $\vec{n}$ depends on $\vec{v}$ and therefore needs to be recalculated every time $\vec{v}$ is updated.

[84] Alternatively, one can also consider differences in the selfenergies.

[85] For details of AMEA and the accuracy expected by taking a certain number $N_{b}$ of bath sites see, e.g., Refs. [63,71,75]. Notice that the accuracy obtained with a certain $N_{b}$ in AMEA is expected 
to be the same or better than with twice the same number of bath sites $\left(2 N_{b}\right)$ in exact diagonalization. Furthermore, the AMEA spectrum is continuous without the need of an artificial broadening.

[86] W. Hofstetter, R. Bulla, and D. Vollhardt, Phys. Rev. Lett. 84, 4417 (2000).

[87] Due to our approximation in the impurity solver, there is a small deviation from current conservation on the correlated bonds. Therefore, our results show the average of the current over the correlated bonds and one of the uncorrelated ones. However, we do not show explicit error-bars since they are within the symbol size of the presented results, Fig. 8.

[88] Results for $U=10$ are not shown because the current density is so low in this case that it lies below our numerical uncertainty.

[89] In principle, this expression is missing the self-energy due to the interaction; however, recall that the Poissonian loop is performed for fixed self-energy and thus this term does not contribute to the sort for the derivative. 